\title{
Luca Fanelli
}

Determinacy, Indeterminacy and Dynamic Misspecification in Linear Rational Expectations Models

\author{
Quaderni di Dipartimento
}

Serie Ricerche 2010, n. 4 ISSN 1973-9346

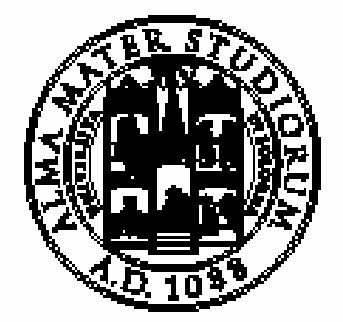

ALMA MATER STUDIORUM UNIVERSITA DI BOLOGNA

Dipartimento di Scienze Statistiche "Paolo Fortunati” 


\title{
Determinacy, Indeterminacy and Dynamic Misspecification in Linear Rational Expectations Models*
}

\author{
Luca Fanelli \\ Quaderni di Dipartimento
}

Serie Ricerche 2010 n. 4

\begin{abstract}
This paper proposes a testing strategy for the null hypothesis that a multivariate linear rational expectations (LRE) model has a unique stable solution (determinacy) against the alternative of multiple stable solutions (indeterminacy). Under a proper set of identification restrictions, determinacy is investigated by a misspecification-type approach in which the result of the overidentifying restrictions test obtained from the estimation of the LRE model through a version of generalized method of moments is combined with the result of a likelihood-based test for the cross-equation restrictions that the LRE places on its finite order reduced form under determinacy. This approach (i) circumvents the nonstandard inferential problem that a purely likelihood-based approach implies because of the presence of nuisance parameters that appear under the alternative but not under the null, (ii) does not involve inequality parametric restrictions and nonstandard asymptotic distributions, and (iii) gives rise to a joint test which is consistent against indeterminacy almost everywhere in the space of nuisance parameters, i.e. except for a point of zero measure which gives rise to minimum state variable solutions, and is also consistent against the dynamic misspecification of the LRE model. Monte Carlo simulations show that the testing strategy delivers reasonable size coverage and power in finite samples. An empirical illustration focuses on the determinacy/indeterminacy of a New Keynesian monetary business cycle model for the US.
\end{abstract}

Keywords: Determinacy, Generalized method of moments, Indeterminacy, LRE model, Identification, Instrumental Variables, Maximum Likelihood, VAR, VARMA.

JEL: C13; C22; E31

${ }^{*}$ I wish to thank Efrem Castelnuovo, Davide delle Monache, Massimo Franchi, Alain Hecq, Marco Lippi, Franz Palm, Franco Peracchi, Tommaso Proietti, Jean-Pierre Urbain and seminar participants at EIEF and Maastricht University for valuable comments and suggestions. The usual disclaimer applies. 


\section{Contents}

1 Introduction 3

$\begin{array}{lll}2 & \text { Intuitions for the testing strategy } & 7\end{array}$

3 Related literature $\quad 14$

4 Structural model and reduced form solutions 16

4.1 Determinate reduced form . . . . . . . . . . . . . . . . . . . 20

4.2 Indeterminate reduced forms $\ldots \ldots \ldots \ldots \ldots \ldots \ldots$

5 Testing strategy 23

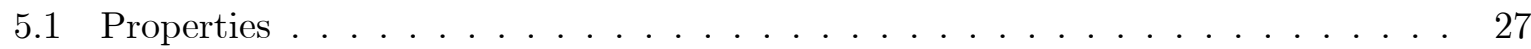

6 Monte Carlo study $\quad 30$

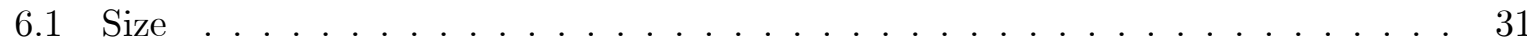

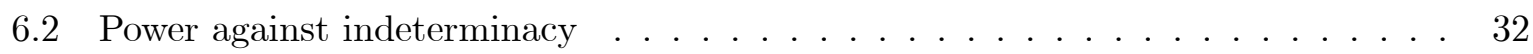

6.3 Power against omitted dynamics . . . . . . . . . . . . . . . . . 33

7 Empirical illustration: a New Keynesian monetary business cycle model 34

8 Concluding remarks $\quad 36$

$\begin{array}{ll}\text { Appendix } & 37\end{array}$

$\begin{array}{ll}\text { Appendix A: Proofs } & 37\end{array}$

Appendix B: Technical supplement $\quad 38$

$.1 \quad$ Estimation issues . . . . . . . . . . . . . . . . . . . . . . . . 39

$.2 \quad$ Further Monte Carlo results . . . . . . . . . . . . . . . . . . . . . . . . 42

$.3 \quad$ Preliminary empirical evidence . . . . . . . . . . . . . . . . . . . . 42

$\begin{array}{ll}\text { Tables } & 35\end{array}$ 


\section{Introduction}

It is well known that linear rational expectations (LRE) models can have multiple equilibria, a situation referred as indeterminacy. Determinacy, on the other hand, denotes a situation in which a LRE model has a unique stable (asymptotically stationary) solution. The time series representation of an indeterminate LRE model may differs substantially from that of a determinate one since a set of nuisance parameters and non-fundamental stochastic disturbances, often referred to as sunspot shocks and usually ascribed to self-fulfilling beliefs of economic agents, ${ }^{1}$ can influence the dynamics of the former.

In dynamic general equilibrium macroeconomic models there are many mechanisms that can generate multiple equilibria such as, among others, strong income effects, incomplete market participation, external increasing returns and monopolistic competition. While many economists interpret indeterminacy as a reflection of reality and a possible explanation of a wide range of economic phenomena (e.g. Benhabib, Farmer, 1999), others argue that it signals coordination problems and affects what could be called the 'predictability' of the system (Guesnerie, 1993). Various selection criteria have been suggested for choosing one among the many possible equilibria, see Driskill (2006) for a recent review.

Recently, macroeconomists have become increasingly interested in evaluating the determinacy/indeterminacy of a particular class of linear(ized) LRE models of the New-Keynesian tradition through which the essential aspects of the business cycle and monetary policy are investigated. Indeed, the equilibrium implied by these models may not be unique if the central bank does not raise sufficiently the nominal interest rate in response to inflation, see e.g. Lubik and Schorfheide (2004), Boivin and Giannoni (2006) and Benati and Surico (2009). This topic, which is currently framed in the debate on the 'Great moderation' has renewed the general interest on the empirical assessment of determinacy/indeterminacy in models involving forward-looking behaviour.

On the econometric side, the non-uniqueness problem in the econometrics of LRE models has traditionally been associated with a particular type of 'explosive indeterminacy' that may arise in models for financial asset markets and foreign exchange markets, or in the Cagan's monetary model of hyperinflation, usually called rational bubbles, see, inter alia, Flood and Garber (1980), Hamilton and Whiteman (1985), West (1987), Casella (1989), Evans (1991), Imrohoroğlu (1993) and Engsted and Nielsen (2010). Aside from rational bubbles, however, only a few of studies, reviewed in detail in Section 3, have dealt with the problem of testing determinacy/indeterminacy in stable LRE models.

In this paper, we address the problem of testing the hypothesis of determinacy in a family

\footnotetext{
${ }^{1}$ These disturbances are also denoted 'rational bubbles' when their occurence give rise to explosive roots.
} 
of multivariate LRE models which covers many of the models currently used in finance and macroeconomics. In this class of models, if a unique stable solution exists, it can be represented, under a proper set of identifying restrictions, as a vector autoregression (VAR) with finite lag order, whose coefficients are subject to nonlinear cross-equation restrictions (CER), see Hansen and Sargent $(1980,1981)$ and Binder and Pesaran $(1995,1997)$. Conversely, if multiple stable solutions occur, these can be represented as vector autoregressive moving average (VARMA)type processes with coefficients subject to (highly) nonlinear CER. These VARMA-type solutions are characterized by two independent sources of indeterminacy which complicate the dynamics and volatility of the system: the former is due by a set of auxiliary parameters that are not related to the structural parameters and enter the MA part of the solution, and, more importantly, are not identifiable under the null of determinacy; the latter is due to the possible presence of sunspot shocks.

Since the VARMA-type solutions obtained under indeterminacy present a richer dynamic structure compared to the VAR solutions obtained under determinacy, the time series properties of the data may lead one to confuse the possible dynamic misspecification of the LRE model (i.e. the omission of lags/leads or variables) with the hypothesis of indeterminacy. According to Lubik and Shorfheide (2004), all system-based approaches to evaluation of indeterminacies are affected by this weakness.

In the current literature, the only formalized model comparison of the hypotheses of determinacy and indeterminacy has been proposed by Lubik and Shorfheide (2004) in the Bayesian framework; other contributions in this area are reviewed in Section 3 which clarifies that no formal test of determinacy is currently available in the classical framework. We attempt to fill this gap by proposing a testing strategy for the null that a multivariate LRE model has a unique stable solution against the alternative of multiple stable solutions which:

(i) does not require prior distributions with the advantage, compared to Lubik and Schorfheide's (2004) approach, that the researcher is exempted from the specification of prior distributions for the arbitrary auxiliary parameters that index the VARMA-type solutions;

(ii) circumvents the nonstandard inferential problem implied by the direct comparison of the likelihood of an highly constrained $\operatorname{VARMA}(p, 0)$ process (determinate solution) with the likelihood of an highly constrained $\operatorname{VARMA}(p+1,0+1)$ process (indeterminate solution); ${ }^{2}$

\footnotetext{
${ }^{2}$ The specific case in which an univariate $\operatorname{ARMA}(0,0)$ process is tested against an ARMA $(1,1)$ process is covered by Hannan (1982) and Andrews and Ploberger (1996), and is generalized to the case of higher-order univariate ARMA models in Veres (1987). Unfortunately, it is difficult to adapt the general solution, discussed in Andrews and Ploberger (1994), to the multivariate framework because of the difficulty in identifying a priori the number of nuisance parameters that index the model solution under indeterminacy, see e.g. Lubik and Schorfheide (2004).
} 
(iii) is not based on the inequality parametric restrictions that identify the determinacy/indeterminacy region of the parameter space, with the advantage that the inference does not require the use of nonstandard asymptotic distributions (Silvapulle and Sen, 2005);

(iv) controls by construction for the possible dynamic misspecification of the LRE model, where the term 'dynamic misspecification' is here used to denote a model that omits relevant lags/leads and/or variables with respect to the data generating process (DGP);

(v) is computationally straightforward and can be implemented with any existing econometric package.

The suggested testing strategy is based on a simple intuition: in a correctly specified LRE model, a test for the hypothesis of determinacy can be formulated as a likelihood-based test for the validity of the CER that the LRE model places on its finite order VAR representation as originally suggested in Hansen and Sargent (1980, 1981); under the maintained assumption of correct specification, the rejection of the CER can be automatically associated with the hypothesis of indeterminacy. This simple argument fails, however, when the correct specification of the LRE model can not be taken for granted; in that case, the rejection of the CER can also be associated with the misspecification of the LRE model.

The idea is to combine in a joint test the outcome of the overidentifying restrictions test applied to the LRE model (Hansen's J-test), with the outcome of a Lagrange multiplier (LM) test for the CER obtained under determinacy. The J-test is used to assess the validity or dynamic misspecification of the LRE model and is obtained from the estimation of the structural parameters by a version of generalized instrumental variables (GIV) (Hansen and Singleton, 1982) based on a finite set of instruments which is directly selected from the reduced form solutions of the model and a parametric estimate of the weighting matrix which accounts for the VMA structure of the LRE model disturbances, see Cumby et al. (1983) and West (1997). Conditional upon the non rejection of the LRE model by the J-test, the LM test for the CER is obtained from the likelihood-based estimation of the constrained unique stable reduced form representation of the LRE model and is used to test its data adequacy. If the CER are not rejected by the LM test the hypothesis of determinacy is accepted and indeterminacy otherwise.

The suggested testing strategy (or joint test), hereafter denoted the ' $\mathrm{J} \rightarrow$ LM' procedure, exploits the merits of both 'limited-' and 'full-information' estimation techniques available for LRE models (Wallis, 1980; Wickens, 1982; West, 1986) and is built upon the following arguments: (a) if in locally identified LRE models 'limited-information' methods are used by applying the same structural identification analysis which is typical of the 'full-information' approach, a finite number of valid over-identifying instruments can be selected in a correctly specified models, and 
generalized method of moments (GMM) (likewise GIV) methods provide consistent estimates of the structural parameters irrespective of whether model solution is determinate or indeterminate; (b) recent research (Mavroeidis, 2005; Jondeau and Le Bihan, 2008) shows that for suitable choices of the weighting matrix, the overidentifying restrictions test resulting from GMM (GIV) estimation has finite sample power against the dynamic misspecification (omission of lags/leads) of the LRE model; (c) since a locally identified and 'correctly specified' LRE model has a finite order VAR representation under determinacy with coefficients subject to a set of nonlinear CER, in a determinate DGP these CER should not be rejected when confronted with the data.

By construction, the asymptotic properties of the joint test inherits, to large extent, the asymptotic properties of the overidentification restrictions and LM tests. It is shown that the overall nominal size can be easily bounded and that in practice one can treat the two tests in the ' $\mathrm{J} \rightarrow \mathrm{LM}$ ' procedure as if they were independent. More importantly, under a set of standard regularity conditions, the ' $\mathrm{J} \rightarrow \mathrm{LM}$ ' procedure is consistent against the hypothesis of indeterminacy almost everywhere in the space of nuisance parameters, i.e. for all values of the nuisance parameters except a zero measure point that generates minimum state variable (MSV) solutions (McCallum, 1983, 2003, 2004) characterized by the same dynamic structure as the determinate reduced form. This property is shared with the Bayesian test proposed by Lubik and Schorfheide (2004) (see their footnote 4). Notably, the joint test is also consistent against the dynamic misspecification of the LRE model and this is a completely novel feature.

Monte Carlo simulations show that the finite sample size coverage of the joint test is satisfactory also for values of the structural parameters which are close to the indeterminacy region of the parameter space. The finite sample power of the joint test is satisfactory for values of the nuisance parameters that are relatively far from the point that generate MSV solutions and, as expected, drops as long as the relative distance of the nuisance parameters from that point decreases.

The implementation of the suggested testing strategy is straightforward and any existing econometric packages with features GMM estimation and/or constrained optimization can be used.

To show the usefulness of the proposed approach, we present an empirical illustration based on a New Keynesian monetary business cycle model of the US economy using the same data set as in Lubik and Schorfheide (2004), who address the same issue by applying their Bayesian approach.

The paper is organized as follows. Section 2 provides a brief account of the concepts of determinacy/indeterminacy by focusing on a simple univariate LRE model and illustrates the intuition behind the suggested testing strategy. Section 3 reviews the econometric literature 
on determinacy/indeterminacy in stable LRE models. Section 4 introduces the multivariate LRE model and derives its reduced forms solutions in the subsections 4.1 and 4.2 , respectively. Section 5 summarizes the suggested testing procedure and discusses it asymptotic properties. Section 6 reports some Monte Carlo evidence on the finite sample performance on the testing procedure and Section 7 provides an empirical illustration based on US data. Section 8 contains some concluding remarks. Proofs are in the Appendix A. Many technical details concerning this paper are summarized in the Appendix B.

\section{Intuitions for the testing strategy}

To fix main ideas, in this section we discuss the determinacy/indeterminacy issue in a simple univariate LRE model, with the objective of providing the intuition underlying the suggested testing strategy. The analysis is extended to the multivariate framework from Section 4 onwards.

Consider the univariate LRE model

$$
X_{t}=\gamma_{f} E_{t} X_{t+1}+\gamma_{b} X_{t-1}+\omega_{t}
$$

in which $X_{t}$ is a scalar, $E_{t} X_{t+1}:=E\left(X_{t+1} \mid \mathcal{I}_{t}\right)$ is the conditional expectation of $X_{t+1}$ upon information $\mathcal{I}_{t}$, and the fundamental structural disturbance $\omega_{t}$ is assumed to obey a martingale difference sequence (MDS) with respect to $\mathcal{I}_{t}\left(E_{t} \omega_{t+1}=0\right)$, and has variance $1.0<\gamma_{f}<1$ and $0 \leq \gamma_{b}<1$ are the structural parameters.

To simplify the discussion, and without loss of generality, assume that the parameter $\gamma_{b}$ is known by the econometrician and fixed at $\gamma_{b}:=\breve{\gamma}_{b}$. A solution to the LRE model is any process $\left\{X_{t}\right\}_{t=0}^{\infty}$ which, for fixed initial condition(s), satisfies Eq. (1). We focus on the class of linear asymptotically stable ARMA-type solutions (Evans and Honkapohja, 1986). Although this class does not cover all possible solutions associated with the LRE model (1), it represents a useful parameterization which is widely used in econometric analysis and for which estimation and testing methods are available.

To rule out the case of explosive solutions, it is conventionally assumed that if $\gamma_{b}:=\breve{\gamma}_{b}=$ $\left(1-\gamma_{f}\right)$ (which implies a unit root in the autoregressive representation of the solution), it holds the restriction $\gamma_{f}<1 / 2$; indeed, with $\breve{\gamma}_{b}=\left(1-\gamma_{f}\right)$ the LRE model in Eq. (1) can be reparameterized in the form

$$
\Delta X_{t}=\frac{\gamma_{f}}{1-\gamma_{f}} E_{t} \Delta X_{t+1}+\left(\frac{1}{1-\gamma_{f}}\right) \omega_{t}
$$

where $\Delta:=(1-L), L$ is the lag operator $\left(L^{j} X_{t}:=X_{t-j}\right)$, and the condition $\gamma_{f}<1 / 2$ ensures that the transformed model has a unique stable solution (see below). With this convention, solutions embodying unit roots can be treated likewise the case of determinacy. 
We maintain, except where explicitly indicated, that the assumption of correct specification holds, i.e. that the DGP belongs to one of the asymptotically stable reduced form solutions associated with Eq. (1).

Given a sample of observations $X_{1}, \ldots, X_{T}$, the null and alternative hypotheses we are interested in are:

$$
\begin{aligned}
& H_{0}: X_{1}, \ldots, X_{T} \text { is generated from the LRE model in Eq. (1) under determinacy } \\
& H_{1}: \quad X_{1}, \ldots, X_{T} \text { is generated from the LRE model in Eq. (1) under indeterminacy. }
\end{aligned}
$$

Before discussing possible ways of testing $H_{0}$ against $H_{1}$, we briefly review the form of the solutions associated with this simple LRE model.

By defining the rational expectations error $\eta_{t}:=X_{t}-E\left(X_{t} \mid \mathcal{I}_{t-1}\right)$ and using the lag operator, the family of linear solutions to Eq. (1) can be represented in the form

$$
\left(\gamma_{f}-L+\breve{\gamma}_{b} L^{2}\right) X_{t}=\gamma_{f} \eta_{t}-L \omega_{t}
$$

By construction, the forecast error $\eta_{t}$ is a MDS with respect to $\mathcal{I}_{t}$ and since $\omega_{t}$ is also a MDS with respect to $\mathcal{I}_{t}$, a linear solution can be also obtained by replacing $\eta_{t}$ with the expression

$$
\eta_{t}:=\kappa \omega_{t}+s_{t}
$$

where $\kappa$ is an arbitrary parameter that can take any value in $\mathbb{R}$ and $s_{t}$ is an extraneous (to the model) variable, often referred to as sunspot shock, such that: (i) $\mathcal{S}_{t}:=\sigma\left(s_{t}, s_{t-1}, \ldots, s_{1}\right) \subset \mathcal{I}_{t}$, (ii) $s_{t}$ is a MDS with respect to $\mathcal{I}_{t}$ with variance $\sigma_{s}^{2}$. By using Eq. (5) in Eq. (4) and re-arranging terms, yields

$$
\left(\gamma_{f}-L+\breve{\gamma}_{b} L^{2}\right) X_{t}=\left(\gamma_{f} \kappa-L\right) \omega_{t}+\gamma_{f} s_{t} ;
$$

it can be easily proved that if Eq. (4) is a solution of model (1), also Eq. (6) is a solution.

Consider for simplicity the case $s_{t}:=0$ a.s. $\forall t$. In this case Eq. (4) collapses to the $\operatorname{ARMA}(2,1)$ model

$$
\left(\gamma_{f}-L+\breve{\gamma}_{b} L^{2}\right) X_{t}=\left(\gamma_{f} \kappa-L\right) \omega_{t}
$$

which involves the unknown parameters $\gamma_{f}$ and $\kappa$. As is known, solution properties of Eq. (7) are governed by the roots $\phi_{c, 1}$ and $\phi_{c, 2}$ of the characteristic equation

$$
\gamma_{f} \phi^{2}-\phi+\breve{\gamma}_{b}=0
$$

The subscript ' $c$ ' for $\phi_{c, 1}$ and $\phi_{c, 2}$ is used to stress that the roots of Eq (8) are 'constrained', i.e. depend on $\gamma_{f}$. In general, for $\breve{\gamma}_{b} \neq 0 \phi_{c, 1}$ and $\phi_{c, 2}$ are linked to the structural parameters by the mapping

$$
\phi_{c, 1}+\phi_{c, 2}=\frac{1}{\gamma_{f}} \quad, \quad \phi_{c, 1} \phi_{c, 2}=\frac{\breve{\gamma}_{b}}{\gamma_{f}}
$$


which shows that the sign and magnitude of $\phi_{c, 1}$ and $\phi_{c, 2}$ depend on the sign and relative magnitude of $\gamma_{f}$ and $\breve{\gamma}_{b}$.

According to the values assumed by $\gamma_{f}$ and $\breve{\gamma}_{b}$, the ARMA process in Eq. (7) admits (I) multiple stable asymptotically stationary solutions (indeterminacy), (II) a unique stable solution (determinacy), (III) unstable solutions (explosive indeterminacy). This paper deals with solutions of type (I) and type (II); it is instructive, however, to briefly the features of solutions of type (III). To simplify the analysis it is conventionally assumed that $\phi_{c, 1}$ and $\phi_{c, 2}$ are real (otherwise $\phi_{c, 2}$ would be the complex conjugate of $\phi_{c, 1}$ ) such that $\phi_{c, 1}<\phi_{c, 2}$.

Multiple stable solutions If $\phi_{c, 1}<1$ and $\phi_{c, 2} \leq 1$, the $\operatorname{ARMA}(2,1)$ model in Eq. (7) defines a stationary process for any value assumed by the auxiliary parameter $\kappa$. The solution is indeterminate. Using Eq. (9), these solutions can be written as

$$
X_{t}=\pi_{1} X_{t-1}-\pi_{2} X_{t-2}+\frac{\kappa}{1-\phi_{c, 1} \gamma_{f}} \omega_{t}-\frac{\phi_{c, 2}}{1-\phi_{c, 1} \gamma_{f}} \omega_{t-1}
$$

where $\pi_{1}:=\left(\phi_{c, 1}+\phi_{c, 2}\right)=\frac{1}{\gamma_{f}}, \pi_{2}:=\phi_{c, 1} \phi_{c, 2}=\frac{\breve{\gamma}_{b}}{\gamma_{f}}$ or, equivalently, in the form

$$
\left(1-\phi_{c, 2} L\right)\left(1-\phi_{c, 1} L\right) X_{t}=\frac{1}{1-\phi_{c, 1} \gamma_{f}}\left(\kappa-\phi_{c, 2} L\right) \omega_{t}
$$

The restrictions

$$
\gamma_{f}+\breve{\gamma}_{b}>1,1 / 2<\gamma_{f}<1
$$

are sufficient for indeterminacy.

II. Unique stable solution. When $\phi_{c, 1} \leq 1$ and $\phi_{c, 2}>1$, the unstable root $\phi_{c, 2}$ can be factored out from the model so that the auxiliary parameter $\kappa$ can be uniquely determined from the structural parameters of the LRE model. The indeterminacy stemming from $\kappa$ disappears and a unique stable (or determinate) solution obtains. To see this, focus on the representation in Eq. (11) and impose the condition that $\phi_{c, 2}$ is also a root of the MA polynomial associated with Eq. (7), i.e. that $\kappa:=1$. If $\phi_{c, 1}<1$ this yields the stable AR process

$$
X_{t}=\phi_{c, 1} X_{t-1}+\left(\frac{1}{1-\gamma_{f} \phi_{c, 1}}\right) \omega_{t}
$$

if $\phi_{c, 1}:=1$ the 'stable' solution is written as

$$
\Delta X_{t}=\left(\frac{1}{1-\gamma_{f}}\right) \omega_{t} .
$$

The condition $\gamma_{f}+\breve{\gamma}_{b} \leq 1$ is sufficient for determinacy. ${ }^{3}$

\footnotetext{
${ }^{3}$ This restriction can be obtained by imposing the condition that the quadratic polynominal in Eq (8) is positive at $\phi:=0$ and negative at $\phi:=1$.
} 
III. Explosive solutions. If $\phi_{c, 1}>1$ and $\phi_{c, 2}>1$, one can at most impose that one among $\phi_{c, 1}$ and $\phi_{c, 2}$ factors out from the model but the other unstable root remains in the autoregressive part of the model. In this case the LRE model in Eq. (1) has no asymptotically stationary solution and the non-uniqueness of solutions corresponds to a form of 'explosive' indeterminacy. Inspection of Eq. (9) reveals that the parametric restrictions

$$
\gamma_{f}+\breve{\gamma}_{b}>1,0<\gamma_{f}<1 / 2
$$

are sufficient for explosiveness. In this paper we do not consider solutions of this type.

Several remarks are in order.

Remark 1 Economists often attribute multiple equilibria to the effect of sunspots shocks alone. Actually, the example above shows that the non-uniqueness phenomenon does not necessarily stem from sunspot shocks; this point is clear from Evans and Honkapohja (1986), Salemi (1986), Pesaran (1987), Broze et al. (1991, 1995), Salemi and Song (1992), Lubik and Schorfheide (2004). The arbitrariness of $\kappa$ and thus of the MA polynomial associated with Eq. (10) is sufficient to generate a multiplicity of solutions. The size (multiplicity) of the solution set is further amplified by the presence of sunspots. Broze and Szafarz (1991) deem the indeterminacy implied by $\kappa$ as 'parametric indeterminacy' and Lubik and Schorfheide (2004, p. 205) as 'indeterminacy without sunspots'. It is seen from Eq. (10) that both $\gamma_{f}$ and $\kappa$ can potentially be estimated from the data. ${ }^{4}$

Remark 2 Relax temporarily the assumption that $\gamma_{b}$ is known to the econometrician. It turns out that $\gamma_{f}, \gamma_{b}$ and $\kappa$ are identified in Eq. (10) (indeterminacy), whereas $\gamma_{b}$ and $\kappa$ are not identifiable from Eq. (12) (determinacy). The fact that $\gamma_{b}$ and $\kappa$ can not be estimated consistently under the null of determinacy leads to a well understood nonstandard inferential problem. To make this point clear, consider the simplified DGP based on $\gamma_{b}:=\breve{\gamma}_{b}:=0$ and $\gamma_{f}>0$; here the only (stable) characteristic root of Eq. (8) is $\phi:=\phi_{c, 1}:=\gamma_{f}^{-1}$ so that for $\gamma_{f} \geq 1$ the solution is indeterminate and takes the form

$$
X_{t}=\phi_{c, 1} X_{t-1}+\kappa \omega_{t}-\phi_{c, 1} \omega_{t-1}
$$

while for $0<\gamma_{f}<1$ the solution is determinate and given by

$$
X_{t}=\omega_{t}
$$

\footnotetext{
${ }^{4}$ This means that when indeterminacy occurs, a 'natural' ex-post criterion to select a particular indeterminate equilibrium is provided by the data, in the sense that one can potentially choose the value $\hat{\kappa}$ which maximizes the likelihood of the observations, as originally argued by Chow (1983).
} 
hence it is observationally equivalent to the (unique) minimum state variable (MSV) solution resulting from Eq. (13) when $\kappa:=1$. If the goal of econometric analysis were pure testing determinacy versus indeterminacy, one should compare the likelihood of the $\operatorname{ARMA}(0,0)$ process in Eq. (14) with the likelihood of the $\operatorname{ARMA}(1,1)$ process in Eq. (13). Since $\kappa$ is unknown, this likelihood comparison is formally equivalent to a test for

$$
H_{0}: \phi_{12}:=0 \text { against } H_{1}: \phi_{12} \neq 0
$$

in the $\operatorname{ARMA}(1,1)$ model

$$
X_{t}=\left(\phi_{11}+\phi_{12}\right) X_{t-1}+v_{t}-\phi_{11} v_{t-1},\left|\phi_{11}+\phi_{12}\right|<1 .
$$

In this kind of situation, the likelihood ratio (LR) or LM statistic for $H_{0}$ should be treated as a function of the nuisance parameters and the test based on the supremum of this function, see e.g. Hannan (1982) and Andrews and Ploberger (1994, 1996). The extension of Andrews and Ploberger's (1994) approach to the multivariate setup is complicated by the difficulty of estimating VARMA processes with highly restricted parametric constraints, in which the dimension of the vector $\kappa$ is not known a priori, see the next sections. The Bayesian solution requires a prior distribution for $\gamma_{f}$ and $\kappa$; for this specific example Lubik and Schorfheide (2004) suggest using the parameterization $\kappa:=1+\kappa^{*}$ and centering the marginal distribution of $\kappa^{*}$ around zero, namely on the MSV solution of Eq. (14). Given the prior $p\left(\gamma_{f}, \kappa^{*}\right)=p_{\gamma}\left(\gamma_{f}\right) p_{\kappa}\left(\kappa^{*}\right)$ and the observations $X_{1}, \ldots, X_{T}$, the posterior distribution $p\left(\gamma_{f}, \kappa^{*} \mid X_{1}, \ldots, X_{T}\right)$ can be opportunely evaluated and the posterior probabilities associated with the determinacy and indeterminacy regions of the parameter space computed.

Remark 3 Let $\Pi(L):=\left(1-\phi_{c, 2} L\right)\left(1-\phi_{c, 1} L\right)$ and $\Xi_{\kappa}(L):=\frac{1}{1-\phi_{c, 1} \gamma_{f}}\left(\kappa-\phi_{c, 2} L\right)$ be the AR and MA polynomials associated with the $\operatorname{ARMA}(2,1)$ process in Eq. (11), respectively. In principle, no restrictions on the polynomial $\Xi_{\kappa}(L)$ are obtained from the LRE model since $\kappa$ is arbitrary and can take any possible real value. In the special case in which $\kappa:=1$, the class of solutions described by Eq. (11) collapses to a reduced form which has the same dynamic structure as the determinate reduced form in Eq. (12). As shown by Evans and Honkapohja (1986), the solutions of the form (16) obtained by deleting common factors, hereafter denoted MSV solutions, are the same as the higher order solutions from which they are obtained, only if the latter satisfy appropriate initial conditions. More precisely, for each stable root of Eq. (8), there will be a MSV solution taking a form similar to that in Eq. (12); to see this, it is sufficient to express the indeterminate solution as

$$
\left(1-\phi_{c, 1} L\right)\left(1-\phi_{c, 2} L\right) X_{t}=\frac{1}{1-\phi_{c, 2} \gamma_{f}}\left(\kappa-\phi_{c, 1} L\right) \omega_{t}
$$


which is obtained from Eq. (11) by exchanging $\phi_{c, 2}$ for $\phi_{c, 1}$; observe that for $\kappa:=1$ this model is the same as that in Eq. (12) but with $\phi_{c, 2}$ replacing $\phi_{c, 1}$. In this example, there are two MSV solutions but in the multivariate context there may exist many MSV solutions with this property (see below). ${ }^{5}$ Suppose now that the DGP belongs to Eq. (11) and $\kappa:=1$ so that the observations are generated by one the two MSV solutions:

$$
X_{t}=\phi_{c, i} X_{t-1}+\left(\frac{1}{1-\gamma_{f} \phi_{c, i}}\right) \omega_{t}, i=1,2
$$

where $\phi_{c, i}, i=1,2$ is a stable root of Eq. (8). A natural question here is: can we recover the 'true' root, say $\phi_{c, *}$, from the data ? The answer is positive, in the sense that the estimation of the autoregressive model in Eq. (16) allows the econometrician to pick out the $\hat{\phi}_{c, *}$ which maximizes the likelihood of the data. ${ }^{6}$

Remark 4 While the process in Eq (10) is causal by construction because $\Pi(z) \neq 0$ for all $z \in \mathbb{R}$ such that $|z| \leq 1$ (Brockell and Davis, 1991, pp. 83-85), the inversion of the MA polynomial $\Xi_{\kappa}(L)$ requires that $\left.|\kappa|>\left|\phi_{c, 2}\right|\right)$. Thus, the parametric indeterminacy can cause nonfundamentalness, in the sense that for $-\phi_{c, 2}<|\kappa|<\phi_{c, 2}$, the inverse autoregressive representation associated with Eq. (10)

$$
a(L) X_{t}=u_{t}
$$

can entail a two-sided polynomial $a(L):=\Xi_{\kappa}(L)^{-1} \Pi(L)$, i.e. involving both positive and negative powers of $L .^{7}$

Remark 5 The determinate solution in Eq. (12) is observationally equivalent to the solution of the model

$$
X_{t}=\gamma_{f} E_{t} X_{t+1} \quad, \quad \gamma_{f}>1
$$

which reads as a very special case of Eq. (1) $\left(\breve{\gamma}_{b}:=0, \omega_{t}:=0\right.$ a.s. $\left.\forall t\right)$. Indeed, Eq. (17) has solution

$$
X_{t}=\frac{1}{\gamma_{f}} X_{t-1}+\eta_{t}
$$

\footnotetext{
${ }^{5}$ Observe that McCallum (2003) suggests considering as unique MSV solution the one that implies $\phi_{c, 1}:=0$ when $\tilde{\gamma}_{b}:=0$.

${ }^{6}$ This consideration suggests that if one imposes the restriction $\gamma_{f} \leq 1-\breve{\gamma}_{b}:=\left(1-\gamma_{f} \phi_{c, 1}\right)$ in the estimation of the LRE model automatically rules out the occurrence of MSV solutions from the solution set.

${ }^{7}$ There are situations in which this issue may have consequences on the selection of valid instruments when the LRE model is estimated by GMM, see Lanne and Saikkonen (2009). However, as is known, one can always replace $\Xi_{\kappa}(L)$ with an equivalent invertible $\mathrm{MA}(1)$ process which has the same autocovariance structure, see e.g. Alessi et al. (2008).
} 
which is indeterminate by construction because $\eta_{t}:=\left(X_{t}-E_{t-1} X_{t}\right)$ is an arbitrary MDS. This is one of the arguments used in Beyer and Farmer $(2003,2004)$ to conclude that it is impossible to decide whether real world data is generated by a determinate or indeterminate LRE model, unless prior restrictions on the dynamics of the model are assumed. Many other examples of this type can be provided. However, using the terminology in Hansen and Sargent (1991), Eq. (17) reads as an 'exact' LRE model while the model with $\omega_{t} \neq 0$ a.s. $\forall t$ is an 'inexact' LRE model. In general, a researcher is interested in knowing whether determinacy/indeterminacy occurs in an 'inexact' LRE model, and not in comparing the reduced forms associated with different classes of structural models.

Remark 6 If $\breve{\gamma}_{b} \neq 0$, the consistent estimation of the parameter $\gamma_{f}$ through 'limited-information' GMM (GIV) methods can be carried out by using e.g. the vector of instruments $Z_{t-1}:=$ $\left(X_{t-1}, X_{t-2}\right)^{\prime}$, which other than being valid and relevant irrespective of whether the LRE model has determinate or indeterminate solution, is over-identifying. Interestingly, Mavroeidis (2005) and Jondeau and Le Bihan (2008) have recently shown that if GIV estimation of LRE models is carried out by using a limited set of instruments and selecting the weighting matrix through West's (1997) parametric procedure, the overidentification restrictions test has reasonable power in samples of typical length.

Suppose we wish to construct a likelihood-based test for $H_{0}$ against $H_{1}$ in Eq.s (2)-(3) (recall that $\gamma_{b}:=\breve{\gamma}_{b}$ is supposed to be known). By comparing Eq. (10) with Eq. (12), it turns out that in this case a LR test for $H_{0}$ against $H_{1}$ requires the comparison of the likelihood of an $\operatorname{ARMA}(1,0)$ process, $L\left(\gamma_{f}, \sigma_{\omega}^{2}\right)$, with the likelihood of an $\operatorname{ARMA}(1+1,0+1)$ process, $L\left(\gamma_{f}, \kappa, \sigma_{\omega}^{2}\right)$. (A similar issue occurs if LM-type tests are considered.) As is known, this problem is nonstandard and can potentially be addressed by use of the asymptotic theory in Veres (1987) and Andrews and Ploberger (1994). This solution, however, becomes prohibitively complicated in the multivariate framework because the estimation of highly restricted VARMA models is cumbersome and the number of auxiliary parameters that index indeterminacy is generally not known a priori (see Section 4). Moreover, the direct comparison of the two likelihoods maintains that the researcher is confident that the LRE model under investigation is correctly specified.

An alternative formulation of the problem might be put forth by substituting $H_{0}$ and $H_{1}$ in Eq.s (2)-(3) with the hypotheses ${ }^{8}$

$$
H_{0}^{\prime}: \gamma_{f} \leq 1-\tilde{\gamma}_{b} \quad, \quad H_{1}^{\prime}: \gamma_{f} \not 1-\tilde{\gamma} .
$$

\footnotetext{
${ }^{8}$ Actually, in order to precisely identify the restrictions that lead to indeterminacy and rule out points of the parameter space that generate e.g. explosive solutions, the 'true' alternative $H_{1}^{\prime}$ should be specified as the intersection of the subsets $\left\{\gamma_{f}, \gamma_{f}>1-\tilde{\gamma}_{b}\right\}$ and $\left\{\gamma_{f}, \gamma_{f}<1 / 2\right\}$.
} 
Necessary condition for a test for $H_{0}^{\prime}$ against $H_{1}^{\prime}$ is the availability of an estimator of $\gamma_{f}$ which is consistent under both hypotheses. The GMM (GIV) estimator of $\gamma_{f}$ is robust to determinacy/indeterminacy (Remark 6) and can potentially be used, but, as is known, any test for $H_{0}^{\prime}$ against $H_{1}^{\prime}$ will have a nonstandard asymptotic distribution, see Silvapulle and Sen (2005, Ch. 4) and references therein. We discuss in Section 4 the complications that make it difficult to extend this approach to the multivariate framework.

A 'classical' testing strategy which rules all problems discussed above out is the objective of the present paper. The idea can be sketched as follows. Under the assumption that the LRE model in Eq. (1) is correctly specified, a test of determinacy might be formulated as a test for the data adequacy of the reduced form in Eq. (12). In principle, one can maximize the likelihood function of this model and apply any available (residual) diagnostic test. Under the assumption of correct specification, the statistical rejection of Eq. (12) as a model that describes the data can automatically be associated with the hypothesis of indeterminacy. When the assumption of correct specification can not be taken for granted, the rejection of the data adequacy of Eq. (12) can be also due to the possible omission of lags/leads and/or relevant variables from the specified LRE model. To account for this type of misspecification, we suggest estimating $\gamma_{f}$ by a version of GMM (GIV) along the lines suggested in Remark 6, and then computing the overidentifying restrictions test..$^{9}$ If the LRE model is not rejected, it makes sense to come back to the analysis of the correct specification of Eq. (12), otherwise it is not possible to conclude whether determinacy or indeterminacy is favoured by the data.

This approach is based on the sequence of two standard misspecification tests; the joint test gives rise to a multiple hypotheses testing issue. This approach will be formalized and extended to the multivariate setup and its properties investigated from Section 4 onwards.

\section{Related literature}

While there exist many contributions in the literature on LRE models on the 'explosive indeterminacy' stemming from rational bubbles, see, inter alia, Flood and Garber (1980), Hamilton and Whiteman (1985), West (1987), Casella (1989), Evans (1991) and Engsted and Nielsen

\footnotetext{
${ }^{9}$ In principle, the difference between the GIV and ML estimates of $\gamma_{f}$ should be statistically negligible under determinacy and statistically significant under indeterminacy (as the ML estimate of $\gamma_{f}$ obtained from Eq. (12) is biased). Therefore, under the assumption of correct specification, a 'natural' test for determinacy against ideterminacy might be based on a Hausman-type test. However, our simulation results show that such an approach poses formidable computational difficulties in finite samples, mainly related to the use of generalized inverses for the difference of the covariance matrices of the two estimators, see Hall (2005, Section 5.5.2) for a detailed explanation. Moreover, if the assumption of correct specifiction of the LRE model is relaxed, the rejection of the null of determinacy by this Hausman-type test could not be ascribed to indeterminacy.
} 
(2010), there are a few of studies which address the econometrics of determinacy/indeterminacy in stable LRE models. ${ }^{10}$ This section reviews the main contributions in which the econometric issues associated with the indeterminacy of multivariate LRE models is more or less explicitly addressed within the context of parametric models.

Jovanovic (1989) and Cooper (2002) discuss the identification and estimation problems that characterize the econometric analysis of models with multiple equilibria from a very general perspective, but it is difficult to relate their analysis to the context of LRE models. To our knowledge, Salemi (1986) is the first article in which it is clearly shown that the nuisance parameters that index the VARMA-type solutions generated by indeterminate LRE models can be estimated consistently by likelihood methods. This point is also clear in Pesaran (1987), Broze and Szafarz (1991) and Salemi and Song (1992), but none of these contributions results in a formalized test for the hypothesis of determinacy.

A likelihood-based test of the hypothesis of determinacy may be found in Imrohoroğlu (1993). This author recognizes that there exist testable parametric restrictions in LRE models which reduce the dimensionality of the multiplicity of solutions. By focusing on a hyperinflation model, he discusses a test for the restrictions that yield a unique (low inflation stationary) equilibrium against the alternative of many (high inflation non-stationary) equilibria, including unstable solutions. A particular feature of Imrohoroğlu's (1993) approach, however, is that the analysis is based on a $\operatorname{VARMA}(2,2)$ model which is treated as the statistical representation of the data and starting point of the analysis. More precisely, all parametric restrictions that lead to determinacy and indeterminacy are derived with respect to the parameters of this $\operatorname{VARMA}(2,2)$ system and none of these leads to e.g. a restricted VARMA $(1,1)$ model. Accordingly, the nonstandard inferential issues that characterize the likelihood-based approach to the problem of testing determinacy in multivariate LRE models are automatically circumvented in Imrohoroğlu's (1993) setup.

A different perspective is provided by Farmer and Guo (1995). According to these authors, while the property of indeterminacy invalidates the 'standard' CER implied by LRE models under determinacy, it gives merit to 'limited-information' estimation techniques which do not require the specification of the implied reduced form. Farmer and Guo (1995) use instrumental variable techniques to estimate the structural parameters of a small-scale business cycle model of the US economy. By using point estimates of the structural parameters, but no inference, they argue that the parametric inequality restrictions that are sufficient for indeterminacy are

\footnotetext{
${ }^{10}$ There exists a remarkable gap between the vast theoretical contributions on indeterminacy, which lead Cochrane (2007, p. 5) to observe that 'Indeterminacy, multiple equilibria, and identification in dynamic rationalexpectations models are huge literatures that I cannot possibly adequately cite, acknowledge, or review in the space of an article', and the papers which face the issue from the econometric point of view.
} 
satisfied in their estimated model. ${ }^{11}$

Binder and Pesaran (1995) discuss a solution method for LRE models in which determinacy is associated with the stability of a quadratic matrix (it corresponds to our $S$ matrix introduced in Section 4), involving nonlinearly the structural parameters; conversely, indeterminacy arises when $S$ has at least one eigenvalue outside the unit circle. They present an empirical application based on the estimation of a real business cycle model of the US economy whose solution uniqueness is checked ex-post by verifying that the eigenvalues of $\hat{S}$, where $\hat{S}$ is the point estimate of $S$, lie inside the unit circle. Although this method for evaluating determinacy is similar in spirit to the informal evaluation method of indeterminacy used in Farmer and Guo (1995), Binder and Pesaran's (1995) approach suggests that a possible solution to the problem of assessing determinacy in LRE models may be that of testing the stability of the $S$ matrix (see the discussion in Section 5). ${ }^{12}$

Finally, Lubik and Schorfheide (2004) provide the Bayesian solution to the problem of assessing the determinacy/indeterminacy of a LRE model. These authors address the issue in the context of New Keynesian monetary business cycle models and put forth a formal model comparison between the two hypotheses, given the data. In particular, using prior distributions for all parameters, including the auxiliary parameters that index equilibria under indeterminacy, they construct probability weights for the determinacy and indeterminacy regions of the parameter space conditional on the observed data (see Remark 2). A crucial issue in Lubik and Schorfheide (2004) analysis, is the specification of the prior distribution for the auxiliary parameters (e.g. the scalar $\kappa$ in the LRE model of Eq. (1)): ingeniously, they recommend centering this prior, when technically feasible, on a MSV solution. Alternatively, they center this prior on one particular solution (the so-called 'baseline' or 'continuity' solution), which is the equilibrium obtained upon the assumption that impulse-response functions to structural shocks do not jump discontinuously at the boundary between the indeterminacy and determinacy regions of the parameter space.

\section{Structural model and reduced form solutions}

In this section we introduce the reference multivariate LRE model and discuss its reduced form solutions.

\footnotetext{
${ }^{11}$ The Monte Carlo experiments in Fanelli (2010) show that 'informal' evaluations of this kind which ignore the uncertainty associated with point estimates may be highly misleading.

${ }^{12}$ For instance, one might apply the distance (Wald-type) test discussed in Kodde and Palm (1987). Other possible tests will be explored in future research.
} 
Let $X_{t}$ the $n \times 1$ vector of observable variables at time $t$. We consider the structural system

$$
\Gamma_{0} X_{t}=\Gamma_{f} E_{t} X_{t+1}+\Gamma_{b} X_{t-1}+\omega_{t}
$$

in which $\Gamma_{i}:=\Gamma_{i}\left(\gamma^{s}\right), i=0, f, b$ are $n \times n$ matrices whose elements depend on the $m_{s} \times 1$ vector of structural parameters $\gamma_{s}, E_{t}:=E\left(\cdot \mid \mathcal{I}_{t}\right), \mathcal{I}_{t}$ is the sigma-field representing the agents' information set and $\omega_{t}$ is the $n \times 1$ fundamental structural disturbance with covariance matrix $\Sigma_{\omega} . X_{0}$ and $X_{-1}$ are treated as non-stochastic at time $t=1$. LRE models in which $\Gamma_{b}:=0_{n \times n}$ are denoted 'purely forward-looking' models.

The structural disturbances $\omega_{t}$ obey an asymptotically stable first-order VAR process

$$
\omega_{t}=R \omega_{t-1}+u_{t}
$$

in which $\omega_{0}$ is fixed, $R$ is a $n \times n$ stable (possibly diagonal) matrix and $u_{t}$ is a MDS with respect to $\mathcal{I}_{t}$ whose covariance matrix $\Sigma_{u}$ fulfils $\Sigma_{u}:=\Sigma_{\omega}-R \Sigma_{\omega} R^{\prime}, \Sigma_{\omega}$ being the covariance matrix of $\omega_{t}$. Throughout the vector of 'truly' structural parameters $\gamma_{s}$ and the non-zero elements of $R$ will be collected in the $m \times 1$ vector $\gamma$ which will be denoted as the vector of structural parameters, with 'true' value $\gamma_{0}$.

The system of Euler equations described by Eq.s (19)-(20) and its equivalent representation in Eq. (21) cover a large class of multivariate LRE models currently used in macroeconomics and finance. More general specifications can be easily accommodated in the setup described by Eq.s (19)-(20) by converting the system to a canonical form, see e.g. Binder and Pesaran (1995) and Appendix B.

A solution to system (19)-(20) is any process $\left\{X_{t}\right\}_{t=0}^{\infty}$ that, for given initial conditions, satisfies the structural equations. Solution properties depend on the location of $\gamma_{0}$ in the space of structural parameters; a crucial issue related to solution properties is the identifiability of $\gamma$, i.e. whether $\gamma$ can uniquely be recovered from the reduced form solution(s) in both the determinacy and indeterminacy regime.

There are situations in which LRE models like in Eq.s (19)-(20) are derived from an underlying agents' optimization problem involving a set of transversality conditions which ensure solution uniqueness, other than stability. In general, however, transversality conditions are not always available, and/or the underlying theory or policy framework upon which the LRE model is derived do not provide enough restrictions on $\gamma$ to rule out multiple equilibria. For instance, the determinacy/indeterminacy of the class of New Keynesian monetary business cycle models discussed from Section 6 onwards may depend, ceteris paribus, on the extent of the response of the monetary policy authority to inflation and output shocks. In these situations, testing whether the observed time series $X_{1}, \ldots, X_{T}$ are generated from a determinate or indeterminate solution of system (19)-(20) is a crucial issue. 
We rewrite the LRE model in Eqs. (19)-(20) in the form

$$
\begin{aligned}
\Gamma_{0}^{R} X_{t}=\Gamma_{f} E_{t} X_{t+1}+\Gamma_{b, 1}^{R} X_{t-1}+\Gamma_{b, 2}^{R} X_{t-2}+u_{t}^{R} \\
\Gamma_{0}^{R}:=\left(\Gamma_{0}+R \Gamma_{f}\right) \\
\Gamma_{b, 1}^{R}:=\left(\Gamma_{b}+R \Gamma_{0}\right) \\
\Gamma_{b, 2}^{R}:=-R \Gamma_{b}
\end{aligned}
$$

such that the 'composite' structural disturbance $u_{t}^{R}:=u_{t}+R \Gamma_{f} \eta_{t}, \eta_{t}:=\left(X_{t}-E_{t-1} X_{t}\right)$ is a MDS with respect to $\mathcal{I}_{t}$. If $\Gamma_{0}$ is non-singular in Eq. (19), $\Gamma_{0}^{R}$ will be non-singular if the matrix $\Gamma_{0}^{-1} R \Gamma_{f}$ has eigenvalues different from -1 . When $R:=0_{n \times n}$ in Eq. (20), system (21) coincides with system (19) and $\omega_{t} \equiv u_{t} \equiv u_{t}^{R}$.

The following assumptions are considered.

Assumption 1 [Stationarity] Given the initial conditions, the solution $\left\{X_{t}\right\}_{t=0}^{\infty}$ associated with the LRE model in Eq.s (19)-(20) belongs to the class of covariance stationary process with $E\left(X_{t}\right)=0_{n \times 1}$.

Assumption 2 [Parameter space] $\gamma_{0}$ is an interior point of the compact space $\mathcal{P} \subset \mathbb{R}^{m}$ and the determinacy region of $\mathcal{P}$ is defined as the sub-set $\mathcal{P}^{D}:=\left\{\gamma \in \mathcal{P}, \xi_{\ell}(\gamma)<0_{\ell \times 1}\right\}$, where $\xi_{\ell}(\gamma)$ is a $\ell$-dimensional differentiable function.

Assumption 3 [Non-singularity] The matrices $\Gamma_{0}, \Gamma_{0}^{R}$ and $\Theta:=\left(\Gamma_{0}^{R}-\Gamma_{f} \Phi_{c, 1}\right)$ are nonsingular, where $\Phi_{c, 1}:=\Phi_{c, 1}(\gamma)$ is a $n \times n$ matrix, defined below, whose elements depend nonlinearly on $\gamma$.

Assumption 4 [Necessary identication condition] $\operatorname{dim}(\gamma):=m \leq 2 n^{2}$.

Assumption 5 [Parameter constancy (within regimes)] $\gamma_{0}$ does not change over the sample $X_{1}, \ldots, X_{T}$.

Assumption 1 rules out non-stationary and explosive processes from the solution set. Unit roots processes can be allowed on condition that system (19)-(20) is interpreted as the stationary equilibrium correction counterpart of a multivariate LRE model involving unit roots; see Fanelli (2010b) for an example.

Assumption 2 establishes that the determinacy region of the parameter space can be identified through a set of $\ell$ inequality constraints involving the structural parameters. It will be shown that in the less favourable case $\xi_{\ell}(\cdot)$ corresponds to a mapping from $\gamma$ to the eigenvalues of a 
matrix (see matrix $S$ below) whose stability is sufficient for determinacy. The indeterminacy region of the parameter space, $\mathcal{P}^{I}$, is a sub-set of $\mathcal{P} \backslash \mathcal{P}^{D}$, i.e. $\mathcal{P}^{I} \subseteq \mathcal{P} \backslash \mathcal{P}^{D}$, because principle $\mathcal{P}$ might include points that generate explosive solutions, conflicting with Assumption 1.

Assumption 3 guarantees that the non-singularity of the matrix of contemporaneous relationships $\Gamma_{0}$ is retained in the $\Gamma_{0}^{R}$ matrix when $R \neq 0_{n \times n}$; the non-singularity of the matrix $\Theta$ is required to represent the equilibria associated with the LRE model as VAR- or VARMA-type processes.

Assumption 4 provides a necessary identification (order) condition and requires the number of structural parameters not to exceed the number of reduced form parameters. ${ }^{13}$

Assumption 5 is motivated by the objective of the testing strategy which is that of assessing whether a given sample of observations is consistent with the hypothesis $\gamma_{0} \in \mathcal{P}^{D}$ or $\gamma_{0} \in \mathcal{P}^{I}$, and not that of detecting possible breakpoints that lead to switches from $\gamma_{0} \in \mathcal{P}^{D}\left(\gamma_{0} \in \mathcal{P}^{I}\right)$ to $\gamma_{0} \in \mathcal{P}^{I}\left(\gamma_{0} \in \mathcal{P}^{D}\right)$

The solutions associated with the multivariate LRE model in Eqs. (19)-(20) are derived in detail in Fanelli (2010b) and are summarized in the next two sub-sections. In this context, solution properties, as well as the local identifiability of $\gamma$ (Rothemberg, 1971) depend on the location of the eigenvalues of the matrix $S:=S(\gamma):=\Theta^{-1} \Gamma_{f}$ with respect to the unit disk. In particular, while determinacy can be associated with the stability of $S$, indeterminacy arises whenever $S$ has at least one eigenvalue outside the unit circle.

Before deriving the determinate and indeterminate reduced forms, we establish sufficient conditions for the local identifiability of the structural parameters.

Proposition 1 [Identification] Let $\mathcal{N}\left(\gamma_{0}\right)$ be a neighborhood of $\gamma_{0}$ in $\mathcal{P}$ and let the $2 n \times 2 n$ matrix

$$
\stackrel{\circ}{\Phi}_{c}:=\stackrel{\circ}{\Phi}_{c}(\gamma):=\left[\begin{array}{cc}
\Phi_{c, 1} & \Phi_{c, 2} \\
I_{n} & 0_{n \times n}
\end{array}\right]
$$

be a stable solution of the quadratic matrix equation

$$
\stackrel{\circ}{\Gamma}_{f}\left(\stackrel{\circ}{\Phi}_{c}\right)^{2}-\stackrel{\circ}{\Gamma}_{0} \stackrel{\circ}{\Phi}_{c}+\stackrel{\circ}{\Gamma}_{b}=0_{2 n \times 2 n}
$$

where

$$
\stackrel{\circ}{\Gamma}_{0}:=\left[\begin{array}{cc}
\Gamma_{0}^{R} & 0_{n \times n} \\
0_{n \times n} & I_{n}
\end{array}\right] \quad, \quad \stackrel{\circ}{\Gamma}_{f}:=\left[\begin{array}{cc}
\Gamma_{f} & 0_{n \times n} \\
0_{n \times n} & 0_{n \times n}
\end{array}\right], \stackrel{\circ}{\Gamma}_{b}:=\left[\begin{array}{cc}
\Gamma_{b, 1}^{R} & \Gamma_{b, 2}^{R} \\
I_{n} & 0_{n \times n}
\end{array}\right]
$$

\footnotetext{
${ }^{13}$ It is worth mentioning that the LRE model introduced in the example of Section 2 does not respect Assumption 4 , irrespective of whether the parameter $\gamma_{b}$ is treated as known or unknown.
} 
and $\Phi_{c, 1}:=\Phi_{c, 1}(\gamma)$ is a component of $S(\gamma):=\left(\Gamma_{0}^{R}-\Gamma_{f} \Phi_{c, 1}\right)^{-1} \Gamma_{f}$. Given the LRE model in Eqs. (19)-(20) and Assumptions 1-5 then (a) if the matrix $S(\gamma)$ is such that

$$
r[S(\gamma)]<1 \quad, \quad \gamma \in \mathcal{N}\left(\gamma_{0}\right)
$$

$r[\cdot]$ being the spectral radius operator, the $a \times 1$ vector $\phi_{c}:=\operatorname{vec}\left[\left(\Phi_{c, 1}: \Phi_{c, 2}\right)\right], a:=(2 n)^{2}$, can be uniquely expressed as function of $\gamma$ in $\mathcal{N}\left(\gamma_{0}\right)$, i.e.

$$
\phi_{c, 0}:=g\left(\gamma_{0}\right)
$$

where $\phi_{c, 0}$ is the 'true' value of $\phi_{c}$ and $g(\cdot)$ a twice differentiable function, and

$$
\operatorname{rank}\left[D\left(\gamma_{0}\right)\right]=m
$$

where

$$
D:=D(\gamma):=\frac{\partial g(\gamma)}{\partial \gamma^{\prime}}
$$

is the $a \times m$ Jacobian matrix associated with $g(\cdot)$; (b) if the matrix $S(\gamma)$ has $0<n_{2} \leq n$ eigenvalues, $\lambda_{2, j}, j=1, \ldots, n_{2}$, that lie outside the unit circle, then a sufficient condition for Eq.s (25)-(26) to hold is that

$$
\varphi_{\phi, h} \lambda_{2, j} \neq 1, h=1, \ldots, 2 n, j=1, \ldots, n_{2}
$$

where $\varphi_{\phi, h}, h=1, \ldots, 2 n$, are the (stable) eigenvalues of the matrix $\stackrel{\circ}{\Phi}_{c}$.

Proof: The proof is a special case of Fanelli (2010b).

\subsection{Determinate reduced form}

Assume that $\gamma_{0} \in \mathcal{P}$ and let $\mathcal{N}\left(\gamma_{0}\right)$ be a neighborhood of $\gamma_{0}$. Under Assumptions 1-5 and the conditions of Proposition 1, if the the LRE model in Eq.s (19)-(20) has a determinate solution, this solution can be represented as the VAR system

$$
X_{t}=\Phi_{c, 1} X_{t-1}+\Phi_{c, 2} X_{t-2}+\Upsilon u_{t}
$$

where $X_{0}$ and $X_{-1}$ are fixed, the $n \times n$ matrices $\Phi_{c, 1}:=\Phi_{c, 1}(\gamma), \Phi_{c, 2}:=\Phi_{c, 2}(\gamma)$ are blocks of the stable companion matrix $\stackrel{\circ}{\Phi}_{c}:=\stackrel{\circ}{\Phi}_{c}(\gamma)$ defined in Eq. (22) and solve Eq. (23); finally, $\Upsilon:=\left(I_{n}-\Theta^{-1} R \Gamma_{f}\right)^{-1} \Theta^{-1} \cdot{ }^{14}$

\footnotetext{
${ }^{14}$ For $\stackrel{\circ}{\Gamma}_{b}:=0_{2 p \times 2 p}$ (purely forward-looking model), the unique stable solution is given by $X_{t}=\Upsilon u_{t}$ unless $\stackrel{\circ}{\Gamma}_{f}$ is invertible and the matrix $\left(\stackrel{\circ}{\Gamma}_{f}\right)^{-1} \stackrel{\circ}{\Gamma}_{0}$ is stable.
} 
Observe that while the stability of $\stackrel{\circ}{\Phi}_{c}$ ensures that the solution is asymptotically stationary, the validity of the condition in Eq. (24), i.e. the local stability of $S(\gamma)$, is sufficient for uniqueness and for the local identifiability of $\gamma$ (part (a) of Proposition 1).

The uniqueness of $\Phi_{c, 1}$ and $\Phi_{c, 2}$ (and $\Upsilon$ ) in $\mathcal{N}\left(\gamma_{0}\right)$ suggests that a consistent estimate of $\gamma$ can be retrieved, under determinacy, from the estimation of the reduced form VAR in Eq.s (28) subject to the restrictions in Eq. (23). These are the CER that the LRE model implies on its determinate reduced form. ${ }^{15}$ Proposition 1 establishes that it is possible to express these CER in explicit form (Eq. (25)), although a closed-form expression for the function $g(\cdot)$ is not readily available.

\subsection{Indeterminate reduced forms}

Assume that $\gamma_{0} \in \mathcal{P}$ and let $\mathcal{N}\left(\gamma_{0}\right)$ be a neighborhood of $\gamma_{0}$. Consider now the case in which, for $\gamma \in \mathcal{N}\left(\gamma_{0}\right)$, the matrix $S:=S(\gamma)$ has $1 \leq n_{2} \leq n$ eigenvalues outside the unit circle. $S(\gamma)$ can be decomposed as

$$
S(\gamma):=P\left[\begin{array}{cc}
\Lambda_{1} & 0_{n_{1} \times n_{2}} \\
0_{n_{2} \times n_{1}} & \Lambda_{2}
\end{array}\right] P^{-1}
$$

where $P:=P(\gamma)$ is $n \times n$ non-singular, $\Lambda_{1}$ is the normal Jordan block that collects the $n_{1}:=n-n_{2}$ eigenvalues that lie inside (or on) the unit circle, and $\Lambda_{2}$ is the normal Jordan block that collects the eigenvalues that lie outside the unit circle. The case in which $S(\gamma)\left(\Lambda_{1}\right)$ has roots on the unit circle is here treated likewise the case of , see the comment following Assumption 1 and Section 2 .

Under Assumptions 1-5, if the LRE model in Eq.s (19)-(20) has multiple stable solutions, these can be represented as the class of VARMA-type processes

$$
X_{t}=\Pi_{1} X_{t-1}+\Pi_{2} X_{t-2}+\Pi_{3} X_{t-3}+\Xi_{0}^{\kappa} u_{t}-\Xi_{1}^{\kappa} u_{t-1}+\tau_{t}
$$

where $X_{0}, X_{-1}$ and $X_{-2}$ are given; the $n \times n$ matrices of autoregressive coefficients $\Pi_{i}, i=1,2,3$ are defined as

$$
\begin{aligned}
& \Pi_{1}:=\left(\Phi_{c, 1}+N_{\Lambda}\right), \\
& \Pi_{2}:=\left(\Phi_{c, 2}-N_{\Lambda} \Phi_{c, 1}\right), \\
& \Pi_{3}:=-N_{\Lambda} \Phi_{c, 2}, \\
& N_{\Lambda}:=P\left[\begin{array}{cc}
0_{n_{1} \times n_{1}} & 0_{n_{1} \times n_{2}} \\
0_{n_{2} \times n_{1}} & \Lambda_{2}^{-1}
\end{array}\right] P^{-1},
\end{aligned}
$$

\footnotetext{
${ }^{15}$ In principle, when technically feasible, the constrained estimation of $\gamma$ should also be based on the imposition of the restriction in Eq. (24).
} 
where $\Phi_{c, 1}$ and $\Phi_{c, 2}$ are $n \times n$ blocks of the stable companion matrix $\stackrel{\circ}{\Phi}_{c}:=\stackrel{\circ}{\Phi}_{c}(\gamma)$ obtained as solution of Eq. (23); the matrices $\Xi_{0}^{\kappa}, \Xi_{1}^{\kappa}$ of moving average coefficients are defined as

$$
\begin{gathered}
\Xi_{0}^{\kappa}:=V_{\kappa} M_{\kappa} \Theta^{-1}, \\
\Xi_{1}^{\kappa}:=N_{\Lambda} M_{\kappa}^{-1} V_{\kappa} M_{\kappa} \Theta^{-1}, \\
M_{\kappa}:=P\left[\begin{array}{cc}
I_{n_{1}} & 0_{n_{1} \times n_{2}} \\
0_{n_{2} \times n_{1}} & \kappa
\end{array}\right] P^{-1}, \operatorname{det}(\kappa) \neq 0, \quad V_{\kappa}:=\left(I_{n}-M_{\kappa} \Theta_{*}^{-1} R \Gamma_{f}\right)^{-1}
\end{gathered}
$$

where $\kappa$ is a $n_{2} \times n_{2}$ matrix containing arbitrary elements (i.e. not related to $\gamma$ ) that, without loss of generality, is here assumed non-singular, and $\Theta:=\left(\Gamma_{0}^{R}-\Gamma_{f} \Phi_{c, 1}\right)$; finally, the stochastic term $\tau_{t}$ is given by

$$
\tau_{t}:=\left[V_{\kappa}-N_{\Lambda} M_{\kappa}^{-1}\left(V_{\kappa}-I_{n}\right) L\right] \xi_{t}
$$

where $\xi_{t}:=\left(0_{n_{1} \times 1}^{\prime}, s_{t}^{\prime}\right)^{\prime}, s_{t}$ is an arbitrary $n_{2} \times 1$ MDS with respect to $\mathcal{I}_{t}$ (sunspot shock) with arbitrary covariance matrix $\Sigma_{s}$.

For future reference, we denote by $\mathcal{K}$ the (open) space of nuisance parameters that index solution multiplicity under indeterminacy, i.e. $\mathcal{K}:=\left\{\operatorname{vec}(\kappa), \operatorname{vec}(\kappa) \in \mathbb{R}^{\left(n_{2}\right)^{2}}\right.$ and $\left.\operatorname{det}(\kappa) \neq 0\right\}$; the non-singularity of $\kappa$ allows us to simplify, without loss of generality, the representation of the indeterminate reduced forms but is not strictly necessary; the non-singularity of $\kappa$ can be relaxed and the resulting equilibria can be represented as shown in Fanelli (2010b).

There are two types of indeterminacies that characterize the VARMA-type reduced forms summarized in Eq.s (30)-(31). First, the presence of the nuisance (auxiliary) parameters in the $\kappa$ matrix which makes the VMA part of the solution arbitrary lead to what we call 'parametric indeterminacy'. The problem is that $\kappa$ is not identified under determinacy, see Eq. (28). Second, the sunspot shocks summarized in $\tau_{t}$ represent an additional source of indeterminacy; when $s_{t}:=0$ a.s. $\forall t\left(\xi_{t}:=0\right.$ a.s. $\forall t$ ('indeterminacy without sunspots'), system (30)-(31) collapses to a truly $\operatorname{VARMA}(3,1)$ process with highly restricted coefficients but the indeterminacy implied by $\kappa$ remains. ${ }^{16}$

Observe that without further restrictions, the matrix $\stackrel{\circ}{\Phi}_{c}:=\stackrel{\circ}{\Phi}_{c}(\gamma)$ which solves Eq. (23) (i.e. the CER) and determines the autoregressive coefficients of system (30) needs not to be unique; if, however, the eigenvalues of $\stackrel{\circ}{\Phi}_{c}$ and the unstable eigenvalues of the matrix $S(\gamma)$ fulfill the condition in Eq. (27), then $\stackrel{\circ}{\Phi}_{c}$ is unique in $\mathcal{N}\left(\gamma_{0}\right)$ and, regardless of the values assumed by the auxiliary parameters $\kappa$, the vector of structural parameters $\gamma$ is locally identifiable (part (b) of Proposition 1).

\footnotetext{
${ }^{16} \mathrm{~A}$ way to rule out sunspots explicitly is to consider only the variables which enter the econometrician's information set, see Hamilton and Whiteman (1985) and Evans and Honkapohja (1986).
} 
Assuming for presentation clarity that $\xi_{t}:=0$ a.s. $\forall t$ and using simple algebra and lag operator techniques, system (30) can be expressed as

$$
\left(I_{n}-N_{\Lambda} L\right)\left(I_{n}-\Phi_{c, 1} L-\Phi_{c, 2} L^{2}\right) X_{t}=\left(M_{\kappa}-N_{\Lambda} L\right) M_{\kappa}^{-1} V_{\kappa} M_{\kappa} \Theta^{-1} u_{t}
$$

and it is seen that in the special case in which $\kappa:=I_{n_{2}}$ (implying $M_{\kappa}:=I_{n}$ ), the indeterminate solutions collapses to the MSV solution

$$
\left(I_{n}-\Phi_{c, 1} L-\Phi_{c, 2} L^{2}\right) X_{t}=\Upsilon u_{t} \quad, \quad \Upsilon:=V_{\kappa} \Theta^{-1}:=\left(I_{n}-\Theta^{-1} R \Gamma_{f}\right)^{-1} \Theta^{-1}
$$

which have the same dynamic structure as the determinate solution in Eq. (28). Thus, for each stable solution $\stackrel{\circ}{\Phi}_{c}:=\stackrel{\circ}{\Phi}_{c}(\gamma)$ of Eq. (23) subject to the restrictions in Eq. (27), there will exist, under indeterminacy, a MSV solution taking the form in Eq. (33) which is obtained for $\kappa:=I_{n_{2}}$.

We observe that under Assumptions 1-5, systems (33) and (28) can actually be regarded as 'almost observationally equivalent'; indeed, while in the former it holds the inequality $r[S(\gamma)]>$ 1 , in the latter it holds the inequality $r[S(\gamma)] \leq 1$, and these conditions can potentially be used to recover the model which addresses the data better.

The highly nonlinear nature of the constraints characterizing the reduced form in Eq (30) suggests that even when $\xi_{t}:=0$ a.s. $\forall t$, the likelihood maximization with respect to $\gamma$, albeit potentially feasible, is computationally cumbersome. Moreover, in the absence of a priori information about the degree of multiplicity, namely on the number of eigenvalues of the $S(\gamma)$ matrix in Eq. (29) that lie outside the unit circle, the investigator needs to make a guess on $n_{2}$, i.e. on the dimension of the space $\mathcal{K}$, prior to estimation.

\section{$5 \quad$ Testing strategy}

Given the multivariate LRE model (19)-(20) under Assumptions 1-5 and the identification conditions of Proposition 1, let $X_{1}, \ldots, X_{T}$ be a sample of $T$ observations. We are interested in the null hypothesis

$$
H_{0}: X_{1}, \ldots, X_{T} \text { is generated from system (28) }
$$

against the alternative

$$
H_{1}: X_{1}, \ldots, X_{T} \text { is generated from system (30) }
$$

on condition that the LRE model is not preliminary rejected by the data.

Provided a (root- $T$ ) asymptotically Gaussian consistent estimate of $\gamma$ is available from the direct estimation of system (19)-(20) (see Fanelli, 2010), a classical test for determinacy can be formulated by testing the stability of the matrix $S(\gamma)$ along the lines of Kodde and Palm (1987) 
who propose a distance (Wald-type) test with has a nonstandard asymptotic distribution. Alternatively, given the definition of the determinacy region of the parameter space in Assumption 2 , one might test the $\ell$ inequality restrictions

$$
\begin{aligned}
& \xi_{\ell}(\gamma)<0_{\ell \times 1} \text { vs } \xi_{\ell}(\gamma) \nless 0_{\ell \times 1} \text {. } \\
& \gamma_{0} \in \mathcal{P}^{D} \quad \gamma_{0} \in \mathcal{P} \backslash \mathcal{P}^{D}
\end{aligned}
$$

For instance, the condition $\xi_{\ell}(\gamma)<0$, where $\xi_{\ell}(\gamma):=\xi_{1}\left(\gamma_{f}\right):=\gamma_{f}+\breve{\gamma}_{b}-1$, is sufficient for determinacy in the simple LRE model discussed in Section 2. The (root- $T$ ) consistent estimate of $\gamma$ can potentially be used to test the null in Eq. (36), formalizing Guo and Farmer's (1995) intuition. However, any statistical test for the inequalities in Eq. (36) has nonstandard asymptotic distributions, see e.g. Kodde and Palm (1986), Wolak (1989, 1991) and Silvapulle and Sen (2005). Moreover, aside from simple multivariate LRE models, it is generally difficult to uniquely map the determinacy condition $r[S(\gamma)]<1$ into a closed form expression for the elements of the function $\xi_{\ell}(\gamma)$, see e.g. Section 6 . Also the approach based on testing the validity of the inequality restrictions in Eq. (36) maintains that the LRE model under investigation is correctly specified.

In this paper we follow a route that shares with the approaches based on testing the stability of the matrix $S(\gamma)$ or the inequalities of Eq. (36), the idea of circumventing the direct estimation of the indeterminate reduced forms and the nuisance parameters that index solution multiplicity; the advantages of our method, however, are that the knowledge of the function $\xi_{\ell}(\gamma)$ and the use of nonstandard asymptotic theory are not needed, and, notably, the assumption of correct specification of the LRE model is not taken for granted.

The method is based on the sequential application of two standard tests that we briefly review separately before discussing the joint testing strategy.

\section{Test 1}

Let $J_{T}$ be the overidentifying restrictions test statistic resulting from the GMM (GIV) estimation of $\gamma$ based on the following ingredients:

\section{Orthogonality conditions}

$$
E\left[e_{t}(\gamma) \otimes Z_{t-1}\right]=0_{n r \times 1} \quad, \quad t=1, \ldots, T
$$

where $e_{t}(\gamma):=\left(\Gamma_{0}^{R} X_{t}-\Gamma_{f} X_{t+1}-\Gamma_{b, 1}^{R} X_{t-1}-\Gamma_{b, 2}^{R} X_{t-2}\right):=u_{t}^{R}-\Gamma_{f} \eta_{t+1}$ is the $n \times 1$ disturbance associated with the representation in Eq. (21) of the LRE model;

Instruments The $r \times 1(r:=2 n)$ vector $Z_{t-1}:=\left(X_{t-1}^{\prime}, X_{t-2}^{\prime}\right)^{\prime}$ containing a set of overidentifying (Assumption 4) instruments regardless of whether model solution is determinate or indeterminate; 


\section{Criterion}

$$
\min _{\gamma}\left(\frac{1}{T} \sum_{t=1}^{T} b_{t}(\gamma)\right)^{\prime} W_{T}\left(\frac{1}{T} \sum_{t=1}^{T} b_{t}(\gamma)\right)
$$

where $b_{t}(\gamma):=\left(e_{t}(\gamma) \otimes Z_{t-1}\right), W_{T}$ is a $n r \times n r$ symmetric positive semidefinite 'weighting' matrix that converges in probability to a symmetric positive definite matrix $W$;

Choice of weighting matrix Given the $\operatorname{VMA}(1)$ structure of $e_{t}(\gamma)$ and of $b_{t}:=b_{t}(\gamma)$, (under fairly general regularity conditions), the 'optimal' choice of $W$ corresponds to the inverse of

$$
\Omega:=V_{0}+\left(V_{1}+V_{1}^{\prime}\right) \quad, \quad V_{i}:=E\left(b_{t} b_{t-i}^{\prime}\right), i=0,1
$$

see e.g. Cumby et al. (1983) or West (1997).

The $J_{T}$ test assesses the correct specification of the LRE model and under Assumptions 1-5 and the hypothesis of correct specification of the LRE model is asymptotically $\chi^{2}\left(c_{1}\right), c_{1}:=$ $n r-m$; conversely, $J_{T}$ is $O_{p}(T)$ if the LRE model omits some lags or leads, see e.g. West (1986) and Hall (2005), Mavroeidis (2005) and Jondeau and Le Bihan (2008). ${ }^{17}$

\section{Test 2}

Let $L M_{T}^{C E R}$ be the LM test statistic for the CER in Eq. (23) obtained from the ML estimation of $\gamma$ from the reduced form VAR solution in Eq.s (28)-(23). As implied by Proposition 1 , the (local) stability of the $S(\gamma)$ matrix is sufficient for estimating $\gamma$ consistently by the maximization of the constrained VAR likelihood, see Appendix B for details. Given the nonlinear nature of the restrictions, under Assumptions 1-5, $L M_{T}^{C E R}$ is $\chi^{2}\left(c_{2}\right)$ with $c_{2}:=2 n^{2}-m$ if the CER hold, and is $O_{p}(T)$ if the CER do not hold, see e.g. Godfrey (1988).

\section{Joint test}

The testing strategy for $H_{0}$ (Eq. (34)) against $H_{1}$ (Eq. (35)) is based on the following sequence:

\footnotetext{
${ }^{17}$ It is well known that, in finite samples, the power of the $J_{T}$ test may be affected by the type of Heteroscedasticity Autocorrelation Covariance (HAC) estimator used for $W$ in Eq. (37) to account for serial correlation and possible heteroscedasticity in the GMM (GIV) residuals. Different HAC estimators, albeit asymptotically equivalent, can differ substantially in finite samples, thus imparting substantial distortions to GMM-based inference (Hall and Inoue, 2003; Hall, 2005). Focusing on LRE models, Mavroeidis (2005) finds that the common practice of using a very large number of instruments, and unnecessarily general corrections for serial correlation, virtually annihilates the power of $J_{T}$ to detect omitted lags in finite samples of order less than 1000; the power of $J_{T}$ can be increased substantially by using fewer instruments and a different weighting matrix which explicitly accounts for the MA structure of disturbances, as outlined above. Similar results are obtained in Jondeau and Le Bihan (2008).
} 
Step 1 Compute the $J_{T}$ test. For fixed $0<\alpha<1$, if $J_{T} \geq \chi_{1-\frac{\alpha}{2}}^{2}\left(c_{1}\right)$, the LRE model is rejected and it does not make sense to investigate its determinacy/indeterminacy; if $J_{T}<\chi_{1-\frac{\alpha}{2}}^{2}\left(c_{1}\right)$ consider the next step.

Step 2 Compute the $L M_{T}^{C E R}$ test. If $L M_{T}^{C E R}<\chi_{1-\frac{\alpha}{2}}^{2}\left(c_{2}\right)$ accept determinacy, otherwise indeterminacy.

Some remarks are in order.

Remark 7 The $L M_{T}^{C E R}$ test can be replaced in the ' $\mathrm{J} \rightarrow \mathrm{LM}$ ' procedure with an alternative, computationally simpler, LM test. Indeed, if the LRE model is correctly specified, the disturbances of the VAR system (28) are uncorrelated under $H_{0}$ and serially correlated under $H_{1}$. Therefore, one may estimate the VAR in Eq. (28) and apply Breush-Godfrey $\mathrm{LM}$ vector test for the absence of residual autocorrelation (AC) against the alternative of residual AC correlation up to order $l \geq 1$ (Hosking, 1981; Brüggemann et al. 2006). Notably, this test can be computed from the estimation of the unrestricted counterpart of the VAR in Eq. (28), i.e. without imposing the CER. ${ }^{18}$ We denote the corresponding test statistic with $L M_{T}^{A C}$, and this statistic is asymptotically $\chi^{2}\left(c_{3}\right), c_{3}:=\ln ^{2}$, under $H_{0}$. The simulation experiments of Section 6 investigate the finite sample performance of the 'J $\rightarrow$ LM' procedure using both $L M_{T}^{C E R}$ and $L M_{T}^{A C}$ in Step 2.

Remak 8 The ' $\mathrm{J} \rightarrow \mathrm{LM}$ ' procedure is computationally straightforward. In practice, fixed the significance level $\alpha$ and obtained the $J_{T}$ test, a practitioner will reject the LRE model if the resulting p-value is less than $\alpha / 2$ (or $\alpha^{*} / 2$, see Remark 11 below), otherwise will estimate the reduced form VAR by ML and compute the $L M_{T}^{C E R}\left(L M_{T}^{A C}\right)$ test. If the p-value associated with the $L M_{T}^{C E R}\left(L M_{T}^{A C}\right)$ test is greater (less) than $\alpha / 2\left(\alpha^{*} / 2\right)$, the hypothesis of determinacy (indeterminacy) is accepted. The $J_{T}$ test has become a standard diagnostic for models estimated by GMM and is routinely calculated in most computer packages; likewise, the $L M_{T}^{A C}$ test is a standard diagnostic for VAR system and is calculated in many computer packages, while the $L M_{T}^{C E R}$ test can be implemented with any econometric package that features (nonlinear) constrained estimation.

Remark 9 The $L M_{T}^{C E R}$ test can be replaced in the Step 2 of the ' $\mathrm{J} \rightarrow$ LM' procedure with a LR test obtained by comparing the unrestricted and constrained likelihoods of the VAR system in Eq. (28). Monte Carlo experiments suggests that in LRE models LR tests can

\footnotetext{
${ }^{18}$ From the computational viewpoint, the key difference between $L M_{T}^{C E R}$ and $L M_{T}^{A C}$ is that the latter can be computed from the VAR in Eq. (28) with or without imposing the CER, whereas the former is based on constrained ML estimation.
} 
be very poorly sized compared to LM tests, see Bekaert and Hodrick (2001). ${ }^{19}$ Likewise, the $L M_{T}^{A C}$ test can potentially be replaced with any vector test for residual AC but recent simulations results show that for relatively small systems and moderately large samples, Breush-Godfrey LM test has a reasonable performance also in systems with highly persistent variables, especially if only low order AC is tested, see Brüggemann et al. (2006).

\subsection{Properties}

This sub-section derives the asymptotic size coverage and power of the ' $\mathrm{J} \rightarrow \mathrm{LM}$ ' procedure.

Let $E_{J}^{\alpha / 2}:=\left\{J_{T}, J_{T} \geq c r_{c_{1}}(\alpha / 2)\right\}$ and $E_{L M}^{\alpha / 2}:=\left\{L M_{T}^{C E R}, L M_{T}^{C E R} \geq c r_{c 2}(\alpha / 2)\right\}$ be the rejection (critical) regions of the two tests comprising the ' $\mathrm{J} \rightarrow$ LM' procedure, where ${ } r_{c_{i}}(\alpha / 2)$ is the $100\left(1-\frac{\alpha}{2}\right)$ percentile of the $\chi^{2}\left(c_{i}\right)$ distribution, $i=1,2 . \bar{E}_{(\cdot)}^{\alpha / 2}$ denotes the corresponding acceptance region. It turns out that

$$
\operatorname{Pr}\left\{\text { reject } H_{0} \mid H_{D G P}\right\}:=\operatorname{Pr}\left\{\left(E_{L M}^{\alpha / 2} \mid \bar{E}_{J}^{\alpha / 2}\right) \cup E_{J}^{\alpha / 2} \mid H_{D G P}\right\}
$$

is the probability of incorrectly rejecting the null of determinacy under a given GDP, conventionally denoted with $H_{D G P}$. In our setup, $H_{D G P}$ will be $H_{0}$ in Eq. (34), or $H_{1}$ in Eq. (35), or will denote a model with respect to which system (19)-(20) is (non-locally) misspecified, see below.

The appealing feature of the probability in Eq. (38) is that $\left(E_{L M}^{\alpha / 2} \mid \bar{E}_{J}^{\alpha / 2}\right)$ and $E_{J}^{\alpha / 2}$ can be treated as disjoint events. Thus, while $\operatorname{Pr}\left\{E_{J}^{\alpha / 2} \mid H_{D G P}\right\}$ depends on the asymptotic behaviour of the $J_{T}$ test under $H_{D G P}$, the probability that the LM test rejects the CER conditional upon the non-rejection of the LRE model by the overidentification restriction test, $\operatorname{Pr}\left\{\left(E_{L M}^{\alpha / 2} \mid \bar{E}_{J}^{\alpha / 2}\right) \mid H_{D G P}\right\}$, can easily be related to the marginal probabilities $\operatorname{Pr}\left\{E_{L M}^{\alpha / 2} \mid H_{D G P}\right\}$ and $\operatorname{Pr}\left\{E_{J}^{\alpha / 2} \mid H_{D G P}\right\}$. As a consequence, the derivation of the size coverage and power of the joint test can be tied to the size and power properties of its two test statistics.

Proposition 2 deals with the asymptotic size.

Proposition 2 [Overall Significance Level] Given the LRE model in Eq.s (19)-(20), Assumptions 1-5 and the hypotheses $H_{0}$ and $H_{1}$ in Eq.s (34)-(35), the ' $\mathrm{J} \rightarrow$ LM' procedure is such that when $H_{D G P}:=H_{0}$,

$$
\operatorname{Pr}\left\{\text { reject } H_{0} \mid H_{0}\right\} \leq \alpha^{*} \quad, \quad T \rightarrow \infty
$$

where $\alpha^{*}:=\alpha+\frac{(\alpha / 2)^{2}}{1-\alpha / 2}$.

\footnotetext{
${ }^{19}$ That for nonlinear restrictions LR $\geq$ LM holds in finite samples is a well known result (Godfrey, 1988, Ch. 2).
} 
Proof: Appendix A.

Fixed $\alpha$ (hence $\alpha^{*}$, Proposition 2) and setting $H_{D G P}:=H_{1}$,

$$
\operatorname{Pr}\left\{\text { reject } H_{0} \mid H_{1}\right\}:=\operatorname{Pr}\left\{\left(E_{L M}^{\alpha / 2} \mid \bar{E}_{J}^{\alpha / 2}\right) \cup E_{J}^{\alpha / 2} \mid H_{1}\right\}
$$

captures the power of the joint test against indeterminacy. In the special case in which the auxiliary parameters that index the VARMA-type solutions take value $\kappa:=I_{n_{2}}$, system (30) collapses to the MSV solution of Eq. (33) and the hypotheses $H_{0}$ and $H_{1}$ become indistinguishable for the ' $\mathrm{J} \rightarrow$ LM' procedure. Proposition 3 shows that the joint test is consistent against $H_{1}$ almost everywhere in the space of auxiliary parameters $\mathcal{K}$.

Proposition 3 [Consistency against indeterminacy] Given the LRE model in Eq.s (19)(20), Assumptions 1-5 and the hypotheses $H_{0}$ and $H_{1}$ in Eq.s (34)-(35), when $H_{D G P}:=H_{1}$

(a) the ' $\mathrm{J} \rightarrow \mathrm{LM}$ ' procedure is such that

$$
\operatorname{Pr}\left\{\text { reject } H_{0} \mid H_{1}\right\} \rightarrow 1 \quad, T \rightarrow \infty
$$

if $\kappa \in \mathcal{K} \backslash\left\{\operatorname{vec}\left(I_{n_{2}}\right)\right\}$; (b) the ' $\mathrm{J} \rightarrow$ LM' procedure is such that

$$
\operatorname{Pr}\left\{\text { reject } H_{0} \mid H_{1}\right\} \rightarrow \alpha^{*} \quad, \quad T \rightarrow \infty
$$

if $\kappa:=I_{n_{2}}$.

Proof: Appendix A.

Finally, in order to evaluate the performance of the ' $\mathrm{J} \rightarrow$ LM' procedure against the possibility that the LRE model omits important propagation mechanisms, we consider the case in which the DGP belongs to a solution of the model

$$
\Gamma_{0}^{R} X_{t}=\Gamma_{f} E_{t} X_{t+1}+\sum_{h=2}^{k_{2}} \Gamma_{f, h} E_{t} X_{t+h}+\Gamma_{b, 1}^{R} X_{t-1}+\Gamma_{b, 2}^{R} X_{t-2}+\sum_{j=3}^{k_{1}} \Gamma_{b, j} X_{t-j}+u_{t}^{R}
$$

which includes, with respect to the specification of Eqs. (19)-(20), $\left(k_{1}-2\right)$ additional lags of $X_{t}$ associated with the matrices $\Gamma_{b, j} \neq 0_{n \times n}, j=3, . ., k_{1},\left(k_{1} \geq 3\right)$, and $\left(k_{2}-1\right)$ additional expectations terms associated with the matrices $\Gamma_{f, h} \neq 0_{n \times n}, h=2, . ., k_{2},\left(k_{2} \geq 2\right)$. The LRE model in Eq. (21) is non-locally misspecified with respect to the 'true' DGP if at least one among the matrices $\Gamma_{b, j}, j=3, . ., k_{1}$ and $\Gamma_{f, h}, h=2, . ., k_{2}$ in Eq. (41) is non-zero.

Let $H_{D G P}:=H_{D M}$ (where 'DM' stands for 'dynamic misspecification') denote the DGP given by one of the possible solutions of the LRE model in Eq. (41); assume further that under $H_{D M}$, Assumptions 1-5 of Section 4 refer to he LRE model in Eq. (41). Fixed $\alpha$ ( $\alpha^{*}$, Proposition 2)

$$
\operatorname{Pr}\left\{\text { reject } H_{0} \mid H_{D M}\right\}=\operatorname{Pr}\left\{\left(E_{L M}^{\alpha / 2} \mid \bar{E}_{J}^{\alpha / 2}\right) \cup E_{J}^{\alpha / 2} \mid H_{D M}\right\}
$$


captures the overall probability of rejecting the null of determinacy under the dynamic misspecification of the LRE model. Proposition 4 shows that the ' $\mathrm{J} \rightarrow \mathrm{LM}$ ' strategy is consistent against $H_{D M}$.

Proposition 4 [Consistency against dynamic misspecification] If $X_{1}, \ldots, X_{T}$ is generated by a solution of the LRE model in Eq. (41) and the ' $\mathrm{J} \rightarrow \mathrm{LM}$ ' procedure is computed with respect to the LRE model in Eq.s (19)-(20), then $\operatorname{Pr}\left\{E_{L M}^{\alpha / 2} \mid H_{D M}\right\} \rightarrow 1$ as $T \rightarrow \infty$ and

$$
\operatorname{Pr}\left\{\text { reject } H_{0} \mid H_{D M}\right\} \rightarrow 1 \text { as } T \rightarrow \infty \text {. }
$$

Proof: Appendix A.

Remark 10 According to Proposition 2, fixed a value for $\alpha^{*}$, the critical values $\chi_{1-\frac{\alpha}{2}}^{2}\left(c_{1}\right)$ and $\chi_{1-\frac{\alpha}{2}}^{2}\left(c_{2}\right)$ associated with the $J_{T}$ and $L M_{T}^{C E R}$ tests can be determined by selecting $\alpha$ as the stable solution to the quadratic equation

$$
\alpha+\frac{(\alpha / 2)^{2}}{1-\alpha / 2}=\alpha^{*}
$$

For instance, with $\alpha^{*}=0.05$ one has $\alpha:=0.049$ whereas with $\alpha:=0.05$ one has $\alpha^{*}=$ 0.05064 , suggesting that in practical applications the quantity $\frac{(\alpha / 2)^{2}}{1-\alpha / 2}$ can be ignored with the consequence that $\alpha$ can be treated as the overall significance level of the joint test.

Remark 11 According to Proposition 3, the ' $\mathrm{J} \rightarrow$ LM' procedure is consistent against indeterminacy 'almost everywhere' in $\mathcal{K}$, i.e. except for a closed set (a point) in $\mathcal{K}$ of zero measure. It turns out that the finite sample power of the ' $\mathrm{J} \rightarrow \mathrm{LM}$ ' procedure will be influenced by the extent of the 'distance' of $\kappa$ from the point $I_{n_{2}}$ as shown by the simulation results of Section 6.

Remark 12 The trivial result of part (a) of Proposition 4 suggests that any test for the existence of a unique stable solution against multiplicity of solutions should be computed on a LRE model that fully captures the dynamics of the data, otherwise the probability of incorrectly selecting the hypothesis of indeterminacy approaches one in the limit.

Remark 13 The asymptotic properties of the ' $\mathrm{J} \rightarrow$ LM' procedure hold irrespective of whether in system (30)-(31) $\tau_{t}:=0$ a.s. $\forall t$ ('indeterminacy without sunspots'), or $\tau_{t} \neq 0$ a.s. $\forall t$ ('indeterminacy with sunspots'). 


\section{Monte Carlo study}

This section reports the results of some Monte Carlo simulations designed to investigate the finite sample properties of the ' $\mathrm{J} \rightarrow \mathrm{LM}$ ' procedure summarized in Section 5 .

Our Monte Carlo experiments are based on a LRE model which is widely used in the macroeconomic literature, i.e. a New Keynesian business cycle monetary model based on the following three equations

$$
\begin{aligned}
y_{t} & =\varpi_{f} E_{t} y_{t+1}+\left(1-\varpi_{f}\right) y_{t-1}-\delta\left(i_{t}-E_{t} \pi_{t+1}\right)+\omega_{y, t} \\
\pi_{t} & =\gamma_{f} E_{t} \pi_{t+1}+\gamma_{b} \pi_{t-1}+\varrho y_{t}+\omega_{\pi, t} \\
i_{t} & =\lambda_{r} i_{t-1}+\left(1-\lambda_{r}\right)\left[\lambda_{\pi} \pi_{t}+\lambda_{y} y_{t}\right]+\omega_{i, t} .
\end{aligned}
$$

$y_{t}, \pi_{t}$ and $i_{t}$ are the output gap, inflation, and the nominal interest rate, respectively; Eq. (42) is an intertemporal IS curve, Eq. (43) is a Phillips curve and Eq. (44) is a policy rule. The vector of structural disturbances $\omega_{t}:=\left(\omega_{y, t}, \omega_{\pi, t}, \omega_{i, t}\right)^{\prime}$ is assumed to obey a VAR processes of the form

$$
\omega_{t}=\rho I_{3} \omega_{t-1}+u_{t} \quad, \quad-1<\rho<1 \quad, \quad u_{t} \sim N\left(0, I_{3}\right) .
$$

We refer to Benati and Surico (2009) (and references therein) for details about the derivations of these three equations and their interpretation. ${ }^{20}$

The attractive feature of the LRE model in Eq.s (42)-(45) is that versions of this system have been estimated (typically by Bayesian methods) to investigate whether US monetary policy has lead to determinacy/indeterminacy over certain historical periods, see Section 7. Another aspect is that the 'standard' or 'generalized Taylor principle' (Woodford, 2003) do not hold in system (42)-(45), unless a proper restrictions on the lag structure and/or the parameters $\varrho, \delta, \varpi_{f}, \gamma_{f}, \gamma_{b}, \lambda_{r}, \lambda_{y}, \lambda_{\pi}$ and $\rho$ is imposed. More precisely, if for instance one considers the specification obtained from system (42)-(45) by setting $\varpi_{f}:=1, \gamma_{f}:=\beta, \gamma_{b}:=0, \lambda_{r}:=0$ and $\rho:=0$ which entail a 'purely forward-looking' model $\left(\Phi_{c, 1}:=0_{3 \times 3}\right)$, it turns out that the vector of 'free' structural parameters is $\gamma_{c}:=\left(\varrho, \delta, \beta, \lambda_{y}, \lambda_{\pi}\right)$, and the stability of the matrix $S\left(\gamma_{c}\right):=\left(\Gamma_{0}-\Gamma_{f} \Phi_{c, 1}\right)^{-1} \Gamma_{f}=\Gamma_{0}{ }^{-1} \Gamma_{f}$, i.e. equilibrium determinacy, can be uniquely associated with the inequality restriction

$$
\xi_{\ell}\left(\gamma_{c}\right):=\xi_{1}\left(\gamma_{c}\right):=\max \left\{\left(1-\frac{1-\beta}{\varrho}\right) \lambda_{y}, 0\right\}-\lambda_{\pi}<0 .
$$

\footnotetext{
${ }^{20}$ Note that the full correspondence with the three-equations model used in Benati and Surico (2009) is obtained by setting $\delta^{-1}:=\sigma$ in the intertemporal IS Eq. (42), $\sigma$ being the elasticity of intertemporal substitution in consumption, and $\gamma_{f}:=\beta /(1+\varkappa \beta)$ and $\gamma_{b}:=\varkappa /(1+\varkappa \beta)$, in the NKPC Eq. (43), $\beta$ being the agents' discount factor and $\varkappa$ a measure of price setters' extent to past inflation.
} 
Eq. (46) can potentially be used to test determinacy along the lines discussed in Section $5 .{ }^{21}$ However, in the absence of the above set of restrictions, it is not generally possible to derive a counterpart of the inequality in Eq. (46) which can profitably be exploited for inference.

Using the notation in Eq.s (19)-(20) and the restriction $\gamma_{b}:=\left(1-\gamma_{f}\right)$, the matrices of structural parameters associated with system (42)-(45) $(n:=3)$ are given by

$\Gamma_{0}:=\left[\begin{array}{ccc}1 & 0 & \delta \\ -\varrho & 1 & 0 \\ -\left(1-\lambda_{r}\right) \lambda_{y} & -\left(1-\lambda_{r}\right) \lambda_{\pi} & 1\end{array}\right], \Gamma_{f}:=\left[\begin{array}{ccc}\varpi_{f} & \delta & 0 \\ 0 & \gamma_{f} & 0 \\ 0 & 0 & 0\end{array}\right], \Gamma_{b}:=\left[\begin{array}{ccc}1-\varpi_{f} & 0 & 0 \\ 0 & \gamma_{b} & 0 \\ 0 & 0 & \lambda_{r}\end{array}\right]$.

In the next two sub-sections we investigate the finite sample performance (empirical overall size and power) of the ' $\mathrm{J} \rightarrow$ LM' procedure using system (42)-(45) as the data generating process. In all experiments $u_{t}$ is generated from the Gaussian distribution $N\left(0_{3 \times 1}, I_{3}\right)$ and samples of length $T=150$ are considered, except where indicated, to mimic situations often encountered in practice.

\subsection{Size}

We consider two DGPs based on two different specifications of the structural parameters $\gamma_{0}$, reported in Table 1 and Table 2, respectively. In the former (Table 1), the data are generated from the determinate VAR solution in Eq.s (28)-(24) by considering a setup in which the 'true' vector $\gamma_{0} \in \mathcal{P}^{D}$ is relatively far from the boundary that separates the determinacy from the indeterminacy region of the parameter space; the metric we use to measure this distance is the spectral radius of the $S\left(\gamma_{0}\right)$ matrix which in this case is equal to $r\left[S\left(\gamma_{0}\right)\right]:=0.91$. In the latter (Table 2), the data are generated by setting $\gamma_{0} \in \mathcal{P}^{D}$ relatively close to $\mathcal{P}^{I}$; in this case $r\left[S\left(\gamma_{0}\right)\right]:=0.99$, and the DGP is obtained from the previous one by simply changing the value of the parameter $\lambda_{\pi}$ in Eq. (44) from 1.5 to 1.03 .

We generated $M=1000$ samples of length $T=150$ from the determinate solution (28)-(23) and applied the ' $\mathrm{J} \rightarrow \mathrm{LM}$ ' procedure to each replication, computing both the $L M_{T}^{C E R}$ and $L M_{T}^{A C}$ test in Step 2. The upper panels of Table 1 and Table 2 report the averages of ML and GIV estimates of $\gamma$ across simulations, along with Monte Carlo standard errors. The lower panels of Table1 and Table 2 report the (marginal) rejection frequencies of the two tests considered separately, and the overall empirical rejection frequency of the joint test; $\alpha$ ( $\alpha^{*}$, see Proposition 1 and Remark 10) is fixed at 0.05 .

\footnotetext{
${ }^{21}$ The condition $\lambda_{\pi}>1+\frac{1-\beta}{\varrho} \lambda_{y}$ resulting from Eq. (46) when $\left(1-\frac{1-\beta}{\varrho}\right) \lambda_{y}>0$, is oftern used to argue that when $\lambda_{\pi}<1$, i.e. monetary policy responds less than proportionality to inflation changes, the 'Taylor principle' is violated.
} 
The empirical rejection frequencies obtained for the (marginal) LM test for the CER confirm that under the correct specification of the LRE model, a test for the data adequacy of the determinate solution amounts to an implicit test for the null of determinacy. More importantly, results show that in both experiments the ' $\mathrm{J} \rightarrow \mathrm{LM}$ ' strategy provides a slightly conservative control of the null of determinacy regardless of the relative distance of $\gamma$ from the indeterminacy region of the parameter space.

\subsection{Power against indeterminacy}

To keep the experiment as simple as possible, the DGP of this section is based on a specification of system (42)-(45) in which $\gamma_{0} \in \mathcal{P}^{I}$, sunspot shocks are absent from the solution set described by Eq.s (30)-(31), i.e. $\tau_{t}:=0$ a.s. $\forall t$ ('indeterminacy without sunspots') and, finally, the matrix $S\left(\gamma_{0}\right)$ has only one eigenvalue outside the unit circle $\left(n_{2}:=1\right)$. In this design, the space of nuisance parameters $\mathcal{K}$ corresponds to $\mathbb{R}$ and the matrix $\kappa$ collapses to a single scalar which governs solutions multiplicity.

The true values of $\gamma$ and $\kappa$ that characterize this DGP, $\gamma_{0}$ and $\kappa_{0}$, respectively, are reported in the upper panel of Table 3; notice that with respect to the DGPs investigated in Table 1 and Table 2 , we only changed the values of the policy parameters $\lambda_{y}$ and $\lambda_{\pi}$. The $S\left(\gamma_{0}\right)$ matrix can be decomposed as

$$
S\left(\gamma_{0}\right):=P\left(\gamma_{0}\right)\left[\begin{array}{ccc}
0 & 0 & 0 \\
0 & 0.286 & 0 \\
0 & 0 & 1.059
\end{array}\right] P^{-1}\left(\gamma_{0}\right)
$$

hence, in light of Eq. (29), $\Lambda_{2}:=1.059=: r\left[S\left(\gamma_{0}\right)\right]$. This experiment depicts a situation in which $\gamma_{0}$ is relatively close to the determinacy region of the parameter space.

The results in Table 3 have been obtained by fixing the nuisance parameter to $\kappa_{0}:=-0.5$; the upper panel of Table 3 reports the averages of ML and GIV estimates of $\gamma$ across simulations along with Monte Carlo standard errors, while the lower panel summarizes the marginal and joint empirical rejection frequencies associated with the ' $\mathrm{J} \rightarrow \mathrm{LM}$ ' procedure. It can be observed that the ML estimates of $\lambda_{y}$ and $\lambda_{\pi}$, which are obtained under the hypothesis of solution determinacy, are biased reflecting the fact that the data have been generated by changing the values of $\lambda_{y}$ and $\lambda_{\pi}$ from the determinate DGPs of Sub-section 6.1. It turns out that in this specific DGP the ' $\mathrm{J} \rightarrow \mathrm{LM}$ ' procedure is powerful against the hypothesis of indeterminacy.

Table 4 investigates the sensitivity of the joint test to the nuisance parameter for fixed $\gamma_{0}$; samples of length $T=150$ and $T=70$ are considered. The range of values chosen for $\kappa$, $\{ \pm 0.5, \pm 0.8,0.99, \pm 15\}$, covers both the case in which $\kappa$ is relatively close to the point $\left(\kappa_{0}:=1\right)$ that generates MSV solutions of the type in Eq. (33), and cases in which $\kappa_{0}$ is relatively far from 
that point. In the chosen DGPs, large values of $\kappa_{0}$ (in absolute value) have the consequence tend to make the magnitude of the elements of the $\Xi_{1}^{\kappa}$ matrix of Eq. (30) comparatively negligible with respect to the magnitude of the parameters in $\Pi_{*, i}, i=1,2,3$.

The results in Table 4 remark that, as expected, the finite sample power of the ' $\mathrm{J} \rightarrow \mathrm{LM}$ ' procedure is satisfactorily for values of the nuisance parameters that are relatively far from the point that generates MSV solutions and tends to decline when $\kappa_{0}$ is close to one. Moreover, the finite sample power of the version of the joint test based on the $L M_{T}^{C E R}$ test statistic is considerably more robust than the version based on the $L M_{T}^{A C}$ statistic to changes in $\kappa_{0}$. Overall, the results in Table 3 and Table 4 suggest that the ' $\mathrm{J} \rightarrow \mathrm{LM}$ ' procedure has reasonable finite sample power against the hypothesis of indeterminacy in correctly specified LRE models.

To sum up, the results of this and the previous sub-section confirm that if the LRE model is correctly specified, a test for determinacy against indeterminacy can be formulated as a test for the validity of the CER that the LRE model entails under determinacy. To fully appreciate the role of the $J_{T}$ test in Step 1, we next consider the power of the joint test against the hypothesis of dynamic misspecification of the system.

\subsection{Power against omitted dynamics}

In order to investigate the power of the ' $\mathrm{J} \rightarrow \mathrm{LM}$ ' procedure against the dynamic misspecification of the LRE model, we consider a DGP in which system (42)-(45) is augmented with an additional lag of $X_{t}$ and an additional structural parameter. More precisely, we generated the data from a version of system (41) in which the matrices $\Gamma_{0}, \Gamma_{f}$ and $\Gamma_{b}$ are the same as those specified in Table 1 , and where $\Gamma_{f, h}:=0_{3 \times 3}$ for $h \geq 2$ and $k_{1}:=3, \Gamma_{b, 3}:=\mu I_{3}$; the 'additional' scalar parameter $\mu$ captures the extend of the (non-local) misspecification. The vector of structural parameters is now given by $\gamma^{*}:=\left(\gamma^{\prime}, \mu\right)^{\prime}$.

Results are summarized in Table 5. The sub-vector $\gamma_{0}$ used in all five DGPs of Table 5 is the same as that in Table 1, while the values chosen for $\mu_{0}$ are reported in the first column of Table 5. In all five experiments, the multivariate LRE model has a unique stable solution.

Table 5 emphasizes that the finite sample power of the ' $\mathrm{J} \rightarrow \mathrm{LM}$ ' procedure against the omission of lags in the LRE model depends, as expected, on the magnitude of $\mu_{0}$, i.e. on the extent of the misspecification. In line with the recent results in Mavroeidis (2005) and Jondeau and Le Bihan (2008), the overidentifying restriction test appears well designed to capture the dynamic misspecification of LRE models if the instruments and weighting matrix are chosen as detailed in Section 5.

One important message from Table 5 is that if the $L M_{T}^{C E R}\left(L M_{T}^{A C}\right)$ test in Step 2 would have been computed by disregarding the outcome of the $J_{T}$ test in Step 1, the misspecification 
of the LRE model would have been systematically and erroneously confused with the hypothesis indeterminacy.

\section{Empirical illustration: a New Keynesian monetary business cycle model}

A vast empirical literature has focused on New Keynesian monetary business cycle models similar to that specified in Eq.s (42)-(44) to investigate possible source(s) of the 'US Great Moderation'22 in attempt to disentangle the relative contributions of two main explanations: 'good policy' and 'good luck', see, inter alia, Clarida et al. (2000), Lubik and Schorfheide (2004), Boivin and Giannoni (2006), Benati and Surico (2009) and Mavroeidis (2010).

A detailed investigation of the 'good policy' and 'good luck' hypotheses goes well beyond the purposes of the present illustration, whose ultimate objective is to show the empirical usefulness of the ' $\mathrm{J} \rightarrow$ LM' procedure. We use the same data as in Lubik and Schorfheide (2004) who focus on the determinacy/indeterminacy of a model similar to that specified in Eq.s (42)-(44), using a Bayesian approach. Data are quarterly and cover the period 1960.q1-1997.q4 and refer to the log real per capita GDP detrended with the Hodrik Prescott filter $\left(y_{t}\right),{ }^{23}$ the inflation rate computed as the annualized percentage change of the CPI-U $\left(\pi_{t}\right)$, and the (annualized) Federal Funds rate in percent $\left(i_{t}\right)$. We split the sample into two sub-samples: the 1960.q1-1979.q2 'Pre-Volcker' period (hereafter Period 1), and the 1979.q3-1997.q4 'Volcker-Greenspan' period (hereafter Period 2).

The estimated version of the New Keynesian monetary business cycle model is based on a version of system (42)-(44) in which $\gamma_{f}:=\beta /(1+\varkappa \beta)$ and $\gamma_{b}:=\varkappa /(1+\varkappa \beta)$, where the discount factor $\beta$ is set at 0.99 (recall that the parameter $\varkappa$ captures price setters' extent to past inflation); moreover, the structural parameters $\delta$ and $\kappa$ have been fixed at the values of column 4 of Table 1 in Benati and Surico (2009), i.e. $\delta:=(2)^{-1}$ and $\kappa:=0.05$, respectively. Finally, we assume that in Eq. (45) the autoregressive coefficients of the three disturbances differ across equations. The unknown parameters are collected in the $8 \times 1$ vector $\gamma:=\left(\varpi_{f}, \varkappa, \lambda_{i}, \lambda_{y}, \lambda_{\pi}, \rho_{i}, \rho_{y}, \rho_{\pi}\right)^{\prime}$.

The upper panel of Table 6 summarizes the GIV and ML estimates of the unknown structural parameters obtained over the two sub-periods and the whole sample. ${ }^{24}$ According to the GIV

\footnotetext{
${ }^{22}$ The dramatic changes in US inflation and output growth volatility observed since the end of the seventies is a phenomenon known in macroeconomics as the 'Great Moderation'.

${ }^{23}$ Results based on a linearly detrended version of the output gap do not change substantially and are available upon request.

${ }^{24}$ From Section 4 it turns out that if the data were generated by system (42)-(45) under determinacy, the reduced form representation of the variables should be consistent with a VAR of lag order two. Appendix B
} 
estimates, the differences between point estimates in the two sub-periods are not striking and seem to involve the autoregressive coefficients of structural disturbances, suggesting a possible change in the persistence of the data. On the other hand, the differences between GIV and ML estimates are marked over each sub-period (as well as over the whole sample), are particularly pronounced for the structural parameter $\varkappa\left(\gamma_{f}\right),{ }^{25}$ and seem in line with a situation in which prevails either indeterminacy or the dynamic misspecification of the system.

The middle panel of Table 6 reports the $\mathrm{J}_{T}$ test obtained from GIV estimation and the $\mathrm{LM}_{T}$ test for the CER obtained under determinacy. It turns out that for a fixed overall significance level of $5 \%$, the joint test leads to the hypothesis of indeterminacy in Period 1 (the p-value associated with the $\mathrm{J}_{T}$ test is well above $\alpha / 2:=0.025$, while the p-value associated with the $\mathrm{LM}_{T}$ test is well below $\left.\alpha / 2\right) .{ }^{26}$ Overall, the results obtained for Period 1 support those in Lubik and Schorfheide (2004) obtained through a Bayesian approach; they also confirm the findings in Clarida et al. (2000) and Boivin and Giannoni (2006) which have been achieved without the use of any formal test.

Focusing on Period 2, the ' $\mathrm{J} \rightarrow \mathrm{LM}$ ' procedure rejects the estimated New Keynesian model (the p-value associated with the $\mathrm{J}_{T}$ test is well below $\alpha / 2$ ), signaling the possible omission of propagation mechanisms. According to our testing strategy, it is impossible to establish unambiguously whether determinacy or indeterminacy prevailed after 1979. If one ignores the result of the $\mathrm{J}_{T}$ test (Step 1) and runs the $\mathrm{LM}_{T}$ test for the CER obtained under determinacy (Step 2), then the hypothesis of indeterminacy is (erroneously) selected. ${ }^{27}$

Overall, the result of our test for Period 2 is in contrast with Lubik and Schorfheide (2004), Clarida et al. (2000) and Boivin and Giannoni (2006) who find evidence in favour of determinacy. Interestingly, our analysis is not completely at odds with the results in Mavroeidis (2010). This author argues that the policy rule coefficients (a counterpart of our policy rule in Eq. (44)) are not sufficiently accurately estimable after 1979 to rule out the possibility of indeterminacy; in brief, using a uni-equational approach and identification-robust methods, Mavroeidis (2010)

reports some prima facie evidence based on the estimation of demeaned VARs of lag orders two and three for $X_{t}:=\left(y_{t}, \pi_{t}, i_{t}\right)^{\prime}$ on both sub-periods. Results shows that for both sub-periods, especially for Period 2, two lags are not sufficient to explain the reduced form dynamics of $X_{t}$; in particular, while a VAR with three lags seems to fit the data satisfactorily in Period 1, more than three lags seems to be required in Period 2.

${ }^{25}$ Jondeau and Le Bihan (2008) discuss in detail the differences between GMM and ML estimates in this class of models.

${ }^{26}$ If the GIV point estimates obtained in Period 1 are treated as 'true' parameter values, the estimated spectral radius of the $S(\gamma)$ matrix turns out to be less then one, i.e. $r[S(\hat{\gamma})]:=0.97$; the Monte Carlo results in Appendix B suggest that outcomes like this are perfectly consistent with the hypothesis indeterminacy.

${ }^{27}$ Furthermore, if the GIV point estimates are treated as 'true' parameter values, the spectral radius of the estimated $S(\gamma)$ matrix turns out to be slightly above one, i.e. $r[S(\hat{\gamma})]:=1.003$; the Monte Carlo results in Appendix $\mathrm{B}$ show that a result like this has to be expected in misspecified LRE models. 
documents the weak identification of the reaction function in Period 2 and provides three possible explanations for this evidence. We can re-interpret Mavroeidis's (2010) result as a consequence of the dynamic misspecification of the whole system (42)-(45) in Period 2.

To conclude this section, we test whether a 'breakpoint' occurred in the structural parameters in 1979q3. We use GIV methods, and following Hall and Sen (1999) consider two possible sources of instability of the structural coefficients: one in which the instability is confined in the parameters alone, and the other in which the instability affects other aspects of the model captured by the over-identification restrictions. The two tests, denoted with $D_{T}$ (which is a LRtype test) and $O_{T}$, respectively, are reported in the lower panel of Table 6 . They indicate that, as expected, the suspected breakpoint does not involve the structural parameters but rather the validity of the overidentifying restrictions alone. This evidence supports the view that a break occurred in 1979q3 that changed the overall dynamic structure of the economy, not only the policy parameters as is commonly argued, strengthening the results obtained by the ' $\mathrm{J} \rightarrow \mathrm{LM}$ ' procedure.

\section{Concluding remarks}

To our knowledge, no classical (frequentist) test of the hypothesis of determinacy has been proposed to date for multivariate LRE models. The only formalized contribution may be found in Lubik and Schorfheide (2004) and is based on a Bayesian approach.

In this paper we attempt to fill this gap by introducing a testing strategy for the hypothesis of determinacy based on the sequential combination of two standard 'diagnostic' tests. The former is the overidentification restrictions test obtained from the estimation of the system of Euler equations comprising the LRE model by a GIV estimator based on a 'minimal' set of over-identifying instruments, which are directly selected from the reduced form solutions, and a parametric estimates of the weighting matrix which accounts for the VMA structure of model disturbances. The latter is a LM test for the CER that the LRE model places on its determinate reduced form, and is obtained from the constrained ML estimation of the finite order VAR representation of the model. If the overidentifying restrictions test does not reject the LRE model, the non rejection of the CER by the LM test is evidence in favor of determinacy, while their rejection can be associated with indeterminacy. Conversely, if the overidentifying restrictions test rejects the LRE model, it is impossible to decide whether the data are favour determinacy or indeterminacy.

The joint test involves a multiple hypothesis testing issue but the typical nonstandard inferential issues that characterize the problem of testing determinacy in LRE models are circumvented. 
Irrespective of whether sunspot shocks are included or not in the indeterminate solutions, the joint test is consistent against indeterminacy almost everywhere in the space of nuisance parameters that index solutions multiplicity; moreover, the joint test is consistent against the dynamic misspecification of the LRE model.

Our Monte Carlo experiments show that in samples of typical length, the joint test provides a substantial control of the overall significance level under determinacy, and delivers good finite sample power against indeterminacy if the auxiliary parameters are sufficiently far from the point that generates MSV solutions. Moreover, the risk of confounding the dynamic misspecification of the LRE model with indeterminacy (or indeterminacy) is under control in finite samples.

We present an empirical illustration in which the hypothesis of determinacy is tested in a prototype New Keynesian monetary business cycle LRE model of the US economy. Our results support the shared view that policy prior the Volcker-Greenspan period had been passive and had opened up the possibility of sunspot fluctuations induced by self-fulfilling expectations. However, the analysis stresses that the estimated LRE model is likely to omit important propagation mechanisms when the Volcker-Greenspan period is considered. It is then impossible to establish unambiguously whether determinacy or indeterminacy prevailed after 1979, unless suitable prior information on the structural parameters is used, or versions of the model that fully address the data are specified.

\section{Appendix}

\section{Appendix A: Proofs}

\section{Proof of Proposition 2}

Under Assumptions 1-5 and with $H_{D G P}:=H_{0}, \operatorname{Pr}\left\{E_{J}^{\alpha / 2} \mid H_{0}\right\} \rightarrow \alpha / 2, \operatorname{Pr}\left\{E_{L M}^{\alpha / 2} \mid H_{0}\right\} \rightarrow$ $\alpha / 2$ as $T \rightarrow \infty$. We note that by construction

$$
\begin{gathered}
\operatorname{Pr}\left\{\text { reject } H_{0} \mid H_{0}\right\}:=\operatorname{Pr}\left\{\left(E_{L M}^{\alpha / 2} \mid \bar{E}_{J}^{\alpha / 2}\right) \cup E_{J}^{\alpha / 2} \mid H_{0}\right\}=\operatorname{Pr}\left\{\left(E_{L M}^{\alpha / 2} \mid \bar{E}_{J}^{\alpha / 2}\right) \mid H_{0}\right\}+\operatorname{Pr}\left\{E_{J}^{\alpha / 2} \mid H_{0}\right\} \\
=\operatorname{Pr}\left\{\left(E_{L M}^{\alpha / 2} \mid \bar{E}_{J}^{\alpha / 2}\right) \mid H_{0}\right\}+\operatorname{Pr}\left\{E_{J}^{\alpha / 2} \mid H_{0}\right\}=\frac{\operatorname{Pr}\left\{\left(E_{L M}^{\alpha / 2} \cap \bar{E}_{J}^{\alpha / 2}\right) \mid H_{0}\right\}}{\operatorname{Pr}\left\{\bar{E}_{J}^{\alpha / 2} \mid H_{0}\right\}}+\operatorname{Pr}\left\{E_{J}^{\alpha / 2} \mid H_{0}\right\} \\
\leq \frac{\operatorname{Pr}\left\{E_{L M}^{\alpha / 2} \mid H_{0}\right\}}{\operatorname{Pr}\left\{\bar{E}_{J}^{\alpha / 2} \mid H_{0}\right\}}+\operatorname{Pr}\left\{E_{J}^{\alpha / 2} \mid H_{0}\right\} \rightarrow \frac{\alpha / 2}{1-\alpha / 2}+\frac{\alpha}{2}=\frac{\alpha}{2}+\frac{\alpha}{2}\left(\frac{1}{1-\alpha / 2}\right) \\
=\frac{\alpha}{2}+\frac{\alpha}{2} \sum_{i=0}^{\infty}\left(\frac{\alpha}{2}\right)^{i}=\frac{\alpha}{2}+\sum_{h=1}^{\infty}\left(\frac{\alpha}{2}\right)^{h}=\frac{\alpha}{2}+\frac{\alpha}{2}+\sum_{h=2}^{\infty}\left(\frac{\alpha}{2}\right)^{h}=\alpha+\frac{(\alpha / 2)^{2}}{1-\alpha / 2} .
\end{gathered}
$$


This completes the proof

\section{Proof of Proposition 3}

(a) Under the Assumptions 1-5 and with $H_{D G P}:=H_{1}, \operatorname{Pr}\left\{E_{J}^{\alpha / 2} \mid H_{1}\right\} \rightarrow \alpha / 2$ and $\operatorname{Pr}\left\{E_{L M}^{\alpha / 2} \mid H_{1}\right\} \rightarrow$ 1 as $T \rightarrow \infty$ if $\kappa \neq I_{n_{2}}$. Thus,

$\operatorname{Pr}\left\{\right.$ reject $\left.H_{0} \mid H_{1}\right\}:=\operatorname{Pr}\left\{\left(E_{L M}^{\alpha / 2} \mid \bar{E}_{J}^{\alpha / 2}\right) \cup E_{J}^{\alpha / 2} \mid H_{1}\right\}=\operatorname{Pr}\left\{\left(E_{L M}^{\alpha / 2} \mid \bar{E}_{J}^{\alpha / 2}\right) \mid H_{1}\right\}+\operatorname{Pr}\left\{E_{J}^{\alpha / 2} \mid H_{1}\right\}$

$$
\begin{gathered}
=\frac{\operatorname{Pr}\left\{\left(E_{L M}^{\alpha / 2} \cap \bar{E}_{J}^{\alpha / 2}\right) \mid H_{1}\right\}}{\operatorname{Pr}\left\{\bar{E}_{J}^{\alpha / 2} \mid H_{1}\right\}}+\operatorname{Pr}\left\{E_{J}^{\alpha / 2} \mid H_{1}\right\} \geq \operatorname{Pr}\left\{\left(E_{L M}^{\alpha / 2} \cap \bar{E}_{J}^{\alpha / 2}\right) \mid H_{1}\right\}+\operatorname{Pr}\left\{E_{J}^{\alpha / 2} \mid H_{1}\right\} \\
\geq\left[1-\operatorname{Pr}\left(\bar{E}_{L M}^{\alpha / 2} \mid H_{1}\right)-\operatorname{Pr}\left(E_{J}^{\alpha / 2} \mid H_{1}\right)\right]+\operatorname{Pr}\left\{E_{J}^{\alpha / 2} \mid H_{1}\right\} \rightarrow 1-\frac{\alpha}{2}+\frac{\alpha}{2}=1
\end{gathered}
$$

where use has been made of the Boole condition. We have thus established that for $T \rightarrow \infty$

$$
\operatorname{Pr}\left\{\text { reject } H_{0} \mid H_{1}\right\} \geq 1
$$

and the result follows accordingly. (b) When $\kappa:=I_{n_{2}}$, system (30) collapses to system (28) hence $H_{1} \equiv H_{0}$ and the result follows from Proposition 2. This completes the proof

\section{Proof of Proposition 4}

$H_{D G P}:=H_{D M}$. The LRE model which respect to which the $L M_{T}^{C E R}$ test is applied is dynamically misspecified hence the CER do not hold and $\operatorname{Pr}\left\{E_{L M}^{\alpha / 2} \mid H_{D M}\right\} \rightarrow 1$ as $T \rightarrow \infty$ (Godfrey, 1988). Since $\operatorname{Pr}\left\{E_{J}^{\alpha / 2} \mid H_{D M}\right\} \rightarrow 1$ as $T \rightarrow \infty$ (Hall, 2005, ch. 4), one has that for $T \rightarrow \infty$

$$
\begin{gathered}
\left.\operatorname{Pr} \text { reject } H_{0} \mid H_{D M}\right\}:=\operatorname{Pr}\left\{\left(E_{L M}^{\alpha / 2} \mid \bar{E}_{J}^{\alpha / 2}\right) \cup E_{J}^{\alpha / 2} \mid H_{D M}\right\} \\
=\operatorname{Pr}\left\{\left(E_{L M}^{\alpha / 2} \mid \bar{E}_{J}^{\alpha / 2}\right) \mid H_{D M}\right\}+\operatorname{Pr}\left\{E_{J}^{\alpha / 2} \mid H_{D M}\right\} \geq \operatorname{Pr}\left\{\left(E_{L M}^{\alpha / 2} \cap \bar{E}_{J}^{\alpha / 2}\right) \mid H_{D M}\right\}+1
\end{gathered}
$$

and the result follows accordingly. This completes the proof

\section{Appendix B: Technical supplement}

This technical supplement is divided into three sub-sections. Sub-section B1 deals with estimation issues. Sub-section B2 integrates the Monte Carlo experiments of Section 6 with further results related to the practice of selecting determinacy/indeterminacy on the basis of point estimates that ignore any uncertainty or inference. Finally, Sub-section B3 integrates the results of Section 7 with some additional empirical evidence. 


\section{$.1 \quad$ Estimation issues}

As is known, LRE models can be estimated either by 'full-' or 'limited-information' methods (Wallis, 1980; Wickens, 1982; West, 1986). With 'full-information' methods, if the LRE model is correctly specified, efficient estimates of the structural parameters are retrieved from the CER that the LRE model places on the implied reduced form(s), hence it is of crucial importance to know whether the solution is determinate or indeterminate. Thus, if a LRE model has an indeterminate (VARMA-type) solution and the researcher mistakenly assumes that the solution is determinate (VAR-type), the estimate of the structural parameters retrieved from the CER will be inconsistent.

With 'limited-information' methods, which amount to GMM (GIV) techniques, estimation involves directly the structural Euler equations of the system. These can be estimated consistently separately or jointly, and only apparently the knowledge of the implied reduced forms is not required, see West (1986), Pesaran (1987), Mavroeidis (2005) and Fuckas and Pagan (2009). In general, limited-information estimates are asymptotically less efficient than the corresponding full-information estimates derived from the 'correct' reduced form but have the merit to be robust to determinacy/indeterminacy. The indiscriminate application of GIV methods in LRE models does not necessarily guarantee that the necessary conditions for identification are met, see Pesaran (1987) and Mavroeidis (2005). However, if limited-information methods are used by applying the same structural identification analysis which is typical of the full-information approach, a finite number of valid over-identifying instruments can be selected in a correctly specified LRE model irrespective of whether it has a determinate or indeterminate solution (see Proposition 3 below for details). In general, the overidentifying restrictions test resulting from GMM (GIV) estimation has power against misspecified models (Hall and Inoue, 2003; Hall 2005; ch. 4).

\section{GIV estimation}

In addition to the details provided in Section 5 , we show that the vector $Z_{t-1}$ is relevant if the LRE model in Eq.s (19)-(20) is correctly specified irrespective of whether it has a unique or multiple stable solutions.

To see this, we first re-write, after proper normalization, the $i$-th equation of system (1) as

$$
X_{i, t}=U_{i, t}^{\prime} \varsigma_{i}\left(\gamma_{i}^{*}\right)+e_{i, t}
$$

where $U_{i, t}:=\left(X_{i, t}^{*^{\prime}}, X_{i, t+1}^{*^{\prime}}, X_{i, t-1}^{*^{\prime}}, X_{i, t-2}^{*^{\prime}}\right)^{\prime}$ is the $c_{i} \times 1$ vector of observable right-hand variables included in the $i$-th equation, $\varsigma_{i}(\cdot)$ is a continuous $c_{i} \times 1$ differentiable vector function whose argument is the is the $m_{i} \times 1\left(m_{i} \leq c_{i}\right)$ vector $\gamma_{i}^{*}$ containing the unrestricted structural parameters entering he $i$-th equation (including the non-zero elements of the $i$-th row of $R$ ). Secondly, 
we show that for $i=1, \ldots, n$, the $r \times c_{i}$ matrix

$$
\Sigma_{Z W, i}:=E\left(Z_{t-1} U_{i, t}^{\prime}\right):=E\left[\left(\begin{array}{c}
X_{t-1} \\
X_{t-2}
\end{array}\right)\left(X_{i, t}^{*^{\prime}}, X_{i, t+1}^{*^{\prime}}, X_{i, t-1}^{*^{\prime}}, X_{i, t-2}^{*^{\prime}}\right)\right]
$$

has full-column rank $c_{i}$ irrespective of whether the LRE model has a determinate or indeterminate solution.

Define $X_{i, t}^{*}:=C_{i}^{0} X_{t}, X_{i, t+1}^{*}:=C_{i}^{f} X_{t+1}, X_{i, t-1}^{*}:=C_{i}^{b_{1}} X_{t-1}$ and $X_{i, t-2}^{*}:=C_{i}^{b_{2}} X_{t-1}$, where $C_{i}^{0}, C_{i}^{f}, C_{i}^{b_{1}}$ and $C_{i}^{b_{2}}$ are selection matrices each containing $n$ columns and whose rows, $c_{i}^{0}, c_{i}^{f}$, $c_{i}^{b_{1}}$ and $c_{i}^{b_{2}}$, respectively, are subject to the constraint $c_{i}:=c_{i}^{0}+c_{i}^{f}+c_{i}^{b_{1}}+c_{i}^{b_{2}} . \Sigma_{Z W, i}$ can be represented as

$$
\Sigma_{Z W, i}:=\left[\begin{array}{llll}
\mathbb{Q}(1) & \mathbb{Q}(2) & \mathbb{Q}(0) & \mathbb{Q}(1) \\
\mathbb{Q}(2) & \mathbb{Q}(3) & \mathbb{Q}(1) & \mathbb{Q}(0)
\end{array}\right]\left[\begin{array}{c}
C_{i}^{0 \prime} \\
C_{i}^{f \prime} \\
C_{i}^{b_{1}^{\prime}} \\
C_{i}^{b_{2} \prime} \\
4 n \times c_{i}
\end{array}\right]
$$

where each $\mathbb{Q}(j):=E\left(X_{t-j} X_{t}^{\prime}\right):=\mathbb{Q}(-j)^{\prime}$ is symmetric and positive definite. Since the last matrix on the right-hand side of Eq. (48) has column rank $c_{i}$, it is sufficient to show that the first matrix on the right-hand side of Eq. (48) has row rank $r:=2 n$. Inspection of the structure of the first matrix on the right-hand side of Eq. (48) shows that irrespective of whether each $\mathbb{Q}(j)$ is computed from system (28) or system (30) (Lütkepohl, 2006), the first block of $n$ rows can not be expressed as linear combinations of the second block of $n$ rows and vice versa. Clearly, this argument holds for each equation $i=1, \ldots, n$ of the system.

\section{ML estimation under determinacy}

In addition to Assumptions 1-5 of Section 4, the ML estimation of $\gamma$ is based upon

Assumption 6 [Gaussian disturbances] The MDS $u_{t}$ in Eq.s (19)-(20) has a multivariate Gaussian distribution, $u_{t} \sim N\left(0, \Sigma_{u}\right)$.

When the data do not comply with Assumption 6, the ML estimator discussed below will be a quasi-maximum likelihood (QML) estimator, and can potentially be asymptotically less efficient than the GMM (GIV) estimator.

The VAR in Eq. (28) can be written as

$$
X_{t}=\Phi(\gamma) Z_{t}+\varepsilon_{t}
$$

where $\Phi(\gamma):=\left[\Phi_{c, 1}: \Phi_{c, 2}\right], \varepsilon_{t}:=\Upsilon(\gamma) u_{t} \sim N\left(0, \Sigma_{\varepsilon}\right), \Sigma_{\varepsilon}:=\Upsilon(\gamma) \Sigma_{u} \Upsilon(\gamma)^{\prime}$ and $\Upsilon(\gamma)=: \Upsilon$, $\Phi_{c, i}(\gamma)=: \Phi_{c, i}, i=1,2$. The notation used in Eq. (49) remarks explicitly the dependence of the 
constrained VAR coefficients on the structural parameters. Observe that $\Phi(\gamma):=H \stackrel{\circ}{\Phi}_{c}(\gamma)$. Let $\log L(\phi)$ be the concentrated log-likelihood function of a VAR of order two corresponding to the unrestricted counterpart of system (49). Under Assumption 6, the concentrated log-likelihood of the constrained VAR is given by

$$
\log L\left(\phi_{c}\right)=C-\frac{T}{2} \log \left[\operatorname{det}\left(\sum_{t=1}^{T}\left(X_{t}-\Phi(\gamma) Z_{t}\right)\left(X_{t}-\Phi(\gamma) Z_{t}\right)^{\prime}\right)\right]
$$

where $C:=-\frac{n T}{2}(\log (2 \pi)+1)$ and $\phi_{c}:=v e[\Phi(\gamma)]$ is the vector of VAR coefficients subject to the restrictions in Eq. (25).

The maximization of the log-likelihood function in Eq. (50) with respect to $\gamma$ can be based on a numerical approximation of the quadratic matrix equation (23). The suggested algorithm can be combined with Quasi-Newton methods as in Fanelli (2010a). Observe that the stability constraint $r[S(\gamma)]<1$ which ensures determinacy is not imposed in estimation, leaving room to the occurrence of MSV solutions. Thus, for values of $\gamma_{0} \in \mathcal{P}^{D}$ close to the indeterminacy region of the parameter space, the estimated matrix $\hat{S}:=S(\hat{\gamma})$ matrix might display eigenvalues outside (or on) the unit circle in relatively small sample sizes. In principle, a number of conditions are known which can be used to give operational content to the restriction $r[S(\gamma)]<1$ without the actual computation of the eigenvalues of this matrix, see e.g. Schoonbeek (1989) and references therein; however, whether these conditions give rise to manageable inequality restrictions that can be easily dealt with in estimation is an issue that has to be determined on a case by case basis. The estimation (and testing) results obtained in Section 6 and Section 7 have been obtained without imposing these constraints.

Given the ML estimate of $\hat{\gamma}$, define $\hat{\phi}_{c}:=\operatorname{vec}\left(\hat{\Phi}_{c}\right)$, and let $X$ and $Z$ be the $T \times 1$ and $T \times 2 n$ matrices of observations of system (49) relative to $X_{t}$ and $Z_{t}$, respectively. The efficient score (LM) statistic for the CER can be written as

$$
L M_{T}:=q\left(\hat{\phi}_{c}\right)^{\prime}\left[\hat{\Sigma}_{\varepsilon} \otimes\left(Z^{\prime} Z\right)\right] q\left(\hat{\phi}_{c}\right)
$$

where

$$
q\left(\hat{\phi}_{c}\right):=\operatorname{vec}\left(\left.\frac{\partial \log L(\phi)}{\partial \phi^{\prime}}\right|_{\phi:=\hat{\phi}_{c}}\right)=\operatorname{vec}\left\{\left[Z^{\prime} X-\left(Z^{\prime} Z\right) \hat{\Phi}_{c}^{\prime}\right]\left(\hat{\Sigma}_{\varepsilon}\right)^{-1}\right\}
$$

is the score of the VAR evaluated at the constrained reduced form estimates $\hat{\phi}_{c}$. Likewise, the LR statistic for the CER is given by

$$
L R_{T}:=-2\left(\log L\left(\hat{\phi}_{c}\right)-\log L(\hat{\phi})\right)
$$

where $\hat{\phi}:=\operatorname{vec}\left(\hat{\Phi}_{u}\right)$ and $\hat{\Phi}_{u}:=\left[\hat{\Phi}_{u, 1}: \hat{\Phi}_{u, 2}\right]$ is the unrestricted ML estimate of the VAR coefficients. Since the CER amount to nonlinear parametric constraints, under the null that the 
CER hold, both $L M_{T}$ and $L R_{T}$ are asymptotically distributed as $\chi^{2}\left(2 n^{2}-m\right)$ and are $O_{p}(T)$ if the CER do not hold, see e.g. Godfrey (1988). The finite sample performance of the $L M_{T}$ test in Eq. (51) is investigated in Section 6.

\section{.2 Further Monte Carlo results}

As shown in Section 4, the stability of the matrix $S(\gamma)$ is sufficient for determinacy in correctly specified LRE models. Moreover, there are cases in which the stability of $S(\gamma)$ can be uniquely associated with as a set of parametric inequality restrictions of the form $\xi_{\ell}(\gamma)<0_{\ell \times 1}$. This fact may tempt one to use the 'robust' point (GIV) estimate of $\gamma, \hat{\gamma}$, to evaluate determinacy by simply inspecting whether the eigenvalues of $S(\hat{\gamma})$ lie inside the unit circle, or whether $\xi_{\ell}(\hat{\gamma})<$ $0_{\ell \times 1}$ (see e.g. Farmer and Guo, 1995).

Evaluations of this type can be highly misleading because of the omission of the uncertainty associated with parameter estimates. In this section we use some Monte Carlo experiments to quantify the risk of incurring in wrong conclusions. We consider some of the DGPs already used in Section 6, and summarize the number of times in which the spectral radius of the estimated matrix $S(\gamma)$ obtained by replacing $\gamma$ with its GIV point estimate $\hat{\gamma}$ is found to be greater than one. Overall results are summarized in Table 7.

The first two rows of Table 7 refer to two determinate DGPs whose only difference is the 'distance' of the 'true' $\gamma_{0}$ from the boundary that separates the determinacy from the indeterminacy regions of the parameter space. Results remark that in 'borderline' situations (second row), the probability of observing point estimates of the eigenvalues of $S(\hat{\gamma})$ that lie outside the unit circle is almost $60 \%$, hence the risk of incorrectly selecting indeterminacy is high.

The third row of Table 7 refers to an indeterminate DGP in which the 'true' value of the nuisance parameter $\kappa$ is very close to the point $\left(\kappa_{0}:=1\right)$ that generates MSV solutions. Surprisingly, in this case the probability of incorrectly selecting determinacy is quite high, i.e. $37 \%$.

The last two rows of Table 7 refer to two DGPs with respect to which the estimated LRE model omits a lag of $X_{t}$. The magnitude of the parameter $\mu_{0}$ quantifies the extent of the misspecification: it turns out that from $50 \%$ to $75 \%$ of cases a researcher who ignores the misspecification of the LRE model will wrongly select indeterminacy on the basis of the inspection of the point estimates of the eigenvalues of $S(\hat{\gamma})$.

\section{$.3 \quad$ Preliminary empirical evidence}

This section complements the empirical illustration sketched in Section 7 of the paper. Table 8 reports some prima facie evidence based on the estimation of a demeaned VAR for $X_{t}:=$ $\left(y_{t}, \pi_{t}, i_{t}\right)^{\prime}$ with 2 and 3 lags, considering the two periods, respectively. 
The results in Table 8 seem to suggest that for both periods, especially for Period 2, two lags are not sufficient to explain the dynamics of $X_{t}$. However, while a VAR with three lags seems to fit the data rather well in Period 1, more than 3 lags are needed to fit the data optimally in Period 2. While these reduced form results seems in line with the idea that indeterminacy might have prevailed in Period 1 (indeed a VAR with three lags might represent the statistical finite-sample approximation of a VARMA-type solution), they are not consistent, as they stand, with the hypothesis that determinacy prevailed in Period 2. Overall, the results in Table 8 can be interpreted by observing that a change might have occurred in the reduced form system for $X_{t}$ from Period 1 to Period 2.

\section{References}

[1] Alessi, L., Barigozzi, M., Capasso, M. (2008), A review of nonfundamentalness and identification in structural VAR models, ECB Working Paper No. 922.

[2] Andrews, D., Ploberger, W. (1994), Optimal tests when a nuisance parameter is present only under the alternative, Econometrica 62, 1383-1414.

[3] Andrews, D., Ploberger, W. (1996), Testing the serial correlation against an $\operatorname{ARMA}(1,1)$ process, Journal of the American Statistical Association 91, 1331-1342.

[4] Benhabib, J., Farmer, R.E.A (1999), Indeterminacy and sunspots in macroeconomics, Handbook of Macroeconomics Vol. 1A, Ch. 6.

[5] Benati, L., Surico, P. (2009), VAR analysis and the Great Moderation, American Economic Review 99, 1636-1652.

[6] Beyer, A., Farmer, R.E.A. (2003), On the indeterminacy of determinacy and indeterminacy, European Central Bank, Working Paper No. 277.

[7] Beyer, A., Farmer, R.E.A. (2004), On the indeterminacy of New-Keynesian economics, European Central Bank, Working Paper No. 323.

[8] Binder, M. and Pesaran, M. H. (1995), Multivariate rational expectations models and macroeconomic modelling: a review and some new results, in Pesaran, M. H. and M. Wickens, eds., Handbook of Applied Econometrics, chap. 3 (Blackwell, Oxford) 139-187.

[9] Binder, M. and Pesaran, M. H. (1997), Multivariate Linear Rational Expectations models, Econometric Theory, 13, 877-888. 
[10] Boivin, J., Giannoni, M.P. (2006), Has monetary policy become more effective ?, Review of Economics and Statistics 88, 445-462.

[11] Brockell, P.J., Davis, R.A. (1991), Time series: theory and methods, Springer (2nd edition).

[12] Broze, L., Gouriéroux, C., Szafarz, A. (1995), Solutions of multivariate rational expectations models, Econometric Theory 11, 229-257.

[13] Broze, L., Szafarz, A. (1991), The econometric analysis of non-uniqueness in rational expectations models, Contribution to Economic Analysis, North Holland Amsterdam.

[14] Brüggemann, R., Lütkepohl, H., Saikkonen, P. (2006), Residual autocorrelation testing for vector error correction models, Journal of Econometrics 134, 579-604.

[15] Casella, A. (1989), Testing for rational bubbles with exogenous or endogenous fundamentals, Journal of Monetary Economics 24, 109-122.

[16] Chow, G.C. (1983), Econometrics, McGraw-Hill, New York.

[17] Clarida, R.J., Galì, J., Gertler, M. (2000), Monetary policy rules and macroeconomic stability: evidence and some theory, Quarterly Journal of Economics 115, 147-180.

[18] Cochrane, J.H. (2007), Determinacy and identification with Taylor rules, NBER Working Paper No. 13410.

[19] Cooper, R. W. (2002), Estimation and identification of structural parameters in the presence of multiple equilibria, Annales D'Économie et Statistique 66, 1-26.

[20] Cumby, R.E., Huizinga, J. and Obstfeld, M. (1983), Two-step two-stage òeast squares estimation in models with rational expectations, Journal of Econometrics 21, 333-355.

[21] Driskill, R. (2006), Multiple equilibria in dynamic rational expectations models: A critical review, European Economic Review 50, 171-210.

[22] Engsted, T., Nielsen, B. (2010), Testing for rational bubbles in a co-explosive vector autoregression, CREATES Research Papers 2010-25.

[23] Evans, G.W. (1991), Pitfalls in testing for explosive bubbles in asset prices, American Economic Review 81, 922-930.

[24] Evans, G., Honkapohja, S. (1986), A complete characterization of ARMA solutions to linear rational expectations models, Review of Economic Studies 53, 227-239. 
[25] Fanelli, L. (2010a), Estimation of Quasi-Rational DSGE monetary models, Working Paper 2009/8, Departement of Statistics, University of Bologna.

[26] Fanelli, L. (2010b), Identification restrictions for determinate and indeterminate Linear Rational Expectations models, Working Paper, University of Bologna.

[27] Farmer, R., Guo, J. (1995), The econometrics of indeterminacy: an applied study, CarnegieRochester Conferences on Public Policy 43, 225-271.

[28] Flood, R.P., Garber, P. (1980), Market fundamentals versus price-level bubbles: the first tests, Journal of Political Economy 8, 745-770.

[29] Fukač, M., Pagan, A. (2009), Limited information estimation and evaluation of DSGE models, Journal of Applied Econometrics 25, 55-70.

[30] Guesnerie, R. (1993), Theoretical tests of the rational expectations hypothesis in economic dynamical models, Journal of Economic Dynamics and Control 17, 847-864.

[31] Hall A.R. (2005), Generalized method of moments, Oxford University Press: Oxford.

[32] Hall, A.R., Inoue, A. (2003), The large sample behaviour of the Generalized Method of Moments estimator in misspecified models, Journal of Econometrics 114, 361-394.

[33] Hall, A.R., Sen, A., Structural stability testing in models estimated by Generalized Method of Moments, Journal of Business and Economic Statistics 17, 335-348.

[34] Hamilton, J., Whiteman, C. (1985), The observable implications of self-fulfilling expectations, Journal of Monetary Economics 16, 353-373.

[35] Hannan, E.J. (1982), Testing for Autocorrelation and Akaike's Criterion, Journal of Applied Probability 19, Essays in Statistical Science, 403-412.

[36] Hansen, L. P. and Sargent, T. J. (1980), Formulating and estimating dynamic linear rational expectations models, Journal of Economic Dynamics and Control 2, 7-46.

[37] Hansen, L. P. and Sargent, T. J. (1981), Linear rational expectations models for dynamically interrelated variables, in Lucas R. E. Jr. and T. J. Sargent (eds.), Rational Expectations and Econometric Practise, Minneapolis: University of Minnesota Press, 127-156.

[38] Hansen, L. P. and Sargent, T. J. (1991), Exact linear rational expectations models: Specification and estimation, in Hansen, L. P and T.J. Sargent (eds.), Rational Expectations Econometrics (Westview Press, Boulder). 
[39] Hansen, L.P., Singleton, K.J. (1982), Generalized instrumental variables estimation of nonlinear rational expectations models, Econometrica 50, 1269-1286.

[40] Higham, N.J., Kim, H-M. (2000), Numerical analysis of a quadratic matrix equation, IMA Journal of Numerical Analysis 20, 499-519.

[41] Hosking, J.R.M. (1981), Lagrange-multiplier tests of multivariate time series models, Journal of the Royal Statistical Society B 43, 219-230.

[42] Imrohoroğlu, S. (1993), testing for sunspot equilibria in the German hyperinflation, Journal of Economic Dynamics and Control 17, 289-317.

[43] Kodde, D.A., Palm, F. C. (1986), Wald criteria for jointly testing equality and ineqality restrictions, Econometrica 54, 1243-1248.

[44] Kodde, D.A., Palm, F. C. (1987), Testing the stability of a linear dynamic model, Statistical Papers 28, 263-270.

[45] Lanne, M., Saikkonen, P. (2009), GMM estimation with noncausal instruments, HECER Discussion Paper No. 274.

[46] Lubik, T. A., Schorfheide, F. (2003), Computing sunpost equilibria in linear rational expectations models, Journal of Economic Dynamics and Control 28, 273-285.

[47] Lubik, T. A., Schorfheide, F. (2004), Testing for indeterminacy: An application to U.S. monetary policy, American Economic Review 94, 190-217.

[48] Lütkepohl, H. (2006), New introduction to multiple time series analysis Springer-Verlag, Berlin.

[49] Jondeau, E., Le Bihan, H. (2008), Examining bias in estimators of linear rational expectations models under misspecification, Journal of Econometrics 143, 375-395.

[50] Jovanovic, B. (1989), Observable implications of models with multiple equilibria, Econometrica 57, 1431-1438.

[51] Mavroeidis, S. (2005), Identification issues in forward-looking models estimated by GMM, with an application to the Phillips curve, Journal of Money Credit and Banking 37, 421-448.

[52] Mavroeidis, S. (2010), Monetary policy rules and macroeconomic stability: some new evidence, American Economic Review, forthcoming. 
[53] McCallum, B. T. (1983), On non-uniqueness in rational expectations models: an attempt at perspective, Journal of Monetary Economics 11, 139-168.

[54] McCallum, B. T. (2003), Multiple-solution indeterminacies in monetary policy analysis, Journal of Monetary Economics 50, 1153-1175.

[55] McCallum, B. T. (2004), On the relationship between determinate and MSV solutions in linear RE models, Economic Letters 84, 55-60.

[56] Pesaran, H.M. (1987), The limits to rational expectations, Basil Blackwell, Oxford.

[57] Rothenberg, T. (1971), Identification in parametric models, Econometrica 39, 577-591.

[58] Salemi, M.K. (1986), Solution and estimation of linear rational expectations models, Journal of Econometrics 31, 41-66.

[59] Schoonbek, L. (1989), Stability and structural forms of economic models, Economic Modelling 6, 182-188.

[60] Silvapulle, M.J., Sen, P.K. (2005), Constrained statistical inference, Wiley.

[61] Uhlig, H. (1999), A Toolkit for analyzing nonlinear dynamic stochastic models easily. In R. Marimon and A. Scott (eds.), Computational methods for the study of dynamics economies, pp. 30-61, Oxford University Press, New York.

[62] Veres, S. (1987), Asymptotic distributions of likelihood ratios for overparameterized ARMA processes, Journal of Time Series Analysis 8, 345-357.

[63] Wallis, K. (1980), Econometric implications of the rational expectations hypothesis, Econometrica 48, 49-73.

[64] West, K.D. (1986), Full- versus limited-information estimation of a rational-expectations model, Journal of Econometrics 33, 367-385.

[65] West, K.D. (1987), A specification test for speculative bubbles, Quarterly Journal of Economics 102, 553-580.

[66] West, K.D. (1997), Another heteroskedasticity- and autocorrelation-consistent covariance matrix estimator, Journal of Econometrics 76, 171-191.

[67] Wickens, M.R. (1982), The efficient estimation of econometric models with rational expectations, Review of Economic Studies 49, 55-67. 
[68] Wolak, F. A. (1989), Testing inequality constraints in linear econometric models, Journal of Econometrics 41, 205-235.

[69] Wolak, F. A. (1989), The local nature of hypothesis tests involving inequality constraints in nonlinear models, Econometrica 59, 981-995. 
Table I. Size of the 'J $\rightarrow$ LM' testing strategy when the data are generated by the LRE model in Eq.s (42)-(45) under determinacy.

\begin{tabular}{lccccccccc}
\hline \hline DGP: structural param. $\gamma_{0}:$ & & & & & & & & \\
$r\left[S\left(\gamma_{0}\right)\right]:=0.91$ & $\varrho$ & $\delta$ & $\varpi_{f}$ & $\gamma_{f}$ & $\lambda_{i}$ & $\lambda_{y}$ & $\lambda_{\alpha}$ & $\rho$ \\
& 0.05 & 0.50 & 0.25 & 0.57 & 0.75 & 0.15 & 1.5 & 0.25 \\
\hline \hline & & & \multicolumn{9}{c}{$T=150$} & & & \\
ML estimates & 0.052 & 0.513 & 0.243 & 0.572 & 0.751 & 0.200 & 1.432 & 0.238 \\
& $(0.011)$ & $(0.071)$ & $(0.043)$ & $(0.033)$ & $(0.040)$ & $(0.125)$ & $(0.345)$ & $(0.060)$ \\
& & & & & & & & \\
GIV estimates & 0.055 & 0.491 & 0.256 & 0.565 & 0.749 & 0.158 & 1.524 & 0.247 \\
& $(0.012)$ & $(0.068)$ & $(0.040)$ & $(0.070)$ & $(0.041)$ & $(0.138)$ & $(0.379)$ & $(0.085)$
\end{tabular}

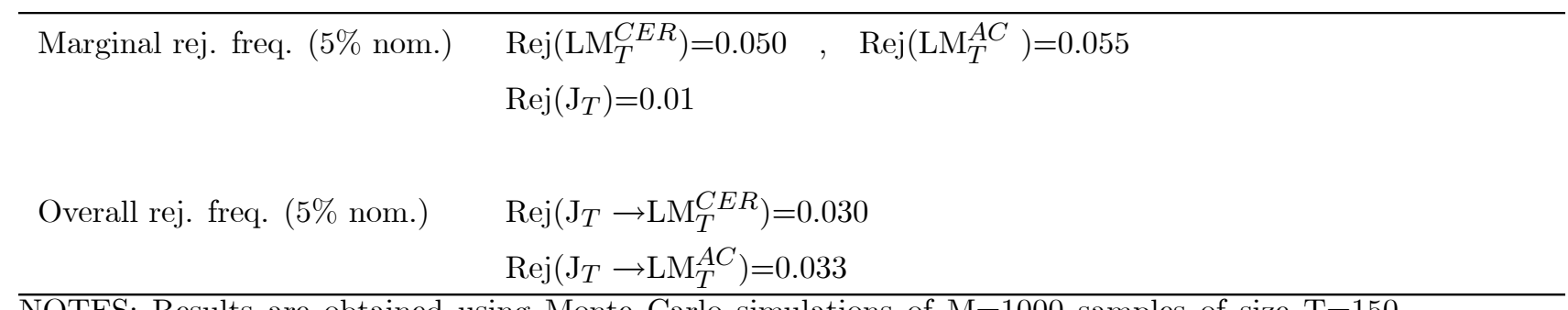

NOTES: Results are obtained using Monte Carlo simulations of $\mathrm{M}=1000$ samples of size $\mathrm{T}=150$.

Each simulated sample is initiated with 100 additional observations to get a stochastic initial state and these are then discarted. UPPER PANEL: ML and GIV estimates of the structural parameters $\gamma$. ML estimates are obtained from the VAR in Eq. (28) subject to the CER in Eq. (23), see Fanelli (2010a). GIV estimates have been computed using $Z_{t-1}:=\left(X_{t-1}^{\prime}, X_{t-2}^{\prime}\right)^{\prime}$ as instruments and a parametric estimate of the weighting matrix to account for the MA(1) disturbances. The MA(1) disturbances in each equation have been approximated by finite order AR processes truncated at lag T/6. Both ML and GIV estimates are obtained by imposing $\lambda_{y}>0, \lambda_{\pi}>0$. Estimates and Monte Carlo standard errors, reported in parentheses, are averages across simulations. LOWER PANEL: Empirical rejection frequencies of the ' $\mathrm{J} \rightarrow \mathrm{LM}$ ' procedure. $\mathrm{J}_{T}$ is the overididentification restrictions test resulting from GIV estimation, $\mathrm{LM}_{T}^{C E R}$ is the LM test for the CER that the LRE model entails under determinacy and $\mathrm{LM}_{T}^{A C}$ is the $\mathrm{LM}$ vector test for the absence of residual $\mathrm{AC}$ against the alternative of correlations up to order one computed from the unrestricted determimate reduced form solution. Rej $(\cdot)$ stands for 'rejection frequency obtained at the $5 \%$ nominal significance level'; $5 \%$ is used for both the marginal tests and the joint test. 


\section{Tables}

Table II. Size of the 'J $\rightarrow$ LM' testing strategy when the data are generated by the LRE model in Eq.s (42)-(45) under determinacy.

\begin{tabular}{|c|c|c|c|c|c|c|c|c|}
\hline $\begin{array}{l}\text { DGP: structural param. } \gamma_{0} \text { : } \\
r\left[S\left(\gamma_{0}\right)\right]:=0.99\end{array}$ & $\begin{array}{c}\varrho \\
0.05 \\
\end{array}$ & $\begin{array}{c}\delta \\
0.50 \\
\end{array}$ & $\begin{array}{l}\varpi_{f} \\
0.25\end{array}$ & $\begin{array}{c}\gamma_{f} \\
0.57 \\
\end{array}$ & $\begin{array}{c}\lambda_{i} \\
0.75 \\
\end{array}$ & $\begin{array}{c}\lambda_{y} \\
0.15 \\
\end{array}$ & $\begin{array}{c}\lambda_{\pi} \\
1.03 \\
\end{array}$ & $\begin{array}{c}\rho \\
0.25 \\
\end{array}$ \\
\hline & \multicolumn{8}{|c|}{$T=150$} \\
\hline ML estimates & $\begin{array}{c}0.052 \\
(0.009)\end{array}$ & $\begin{array}{c}0.508 \\
(0.059)\end{array}$ & $\begin{array}{c}0.246 \\
(0.036)\end{array}$ & $\begin{array}{c}0.574 \\
(0.030)\end{array}$ & $\begin{array}{c}0.742 \\
(0.038)\end{array}$ & $\begin{array}{c}0.208 \\
(0.120)\end{array}$ & $\begin{array}{c}0.896 \\
(0.331)\end{array}$ & $\begin{array}{c}0.241 \\
(0.059)\end{array}$ \\
\hline GIV estimates & $\begin{array}{c}0.055 \\
(0.010)\end{array}$ & $\begin{array}{c}0.493 \\
(0.057)\end{array}$ & $\begin{array}{c}0.255 \\
(0.034)\end{array}$ & $\begin{array}{c}0.567 \\
(0.066)\end{array}$ & $\begin{array}{c}0.744 \\
(0.037)\end{array}$ & $\begin{array}{c}0.201 \\
(0.111)\end{array}$ & $\begin{array}{c}0.921 \\
(0.315)\end{array}$ & $\begin{array}{c}0.245 \\
(0.080)\end{array}$ \\
\hline Marginal rej. freq. $5 \%$ nom. & \multicolumn{8}{|c|}{$\operatorname{Rej}\left(\mathrm{LM}_{T}^{C E R}\right)=0.086 \quad, \quad \operatorname{Rej}\left(\mathrm{LM}_{T}^{A C}\right)=0.052$} \\
\hline Overall rej. freq. $5 \%$ nom. & \multicolumn{8}{|c|}{$\operatorname{Rej}\left(\mathrm{J}_{T} \rightarrow \mathrm{LM}_{T}^{C E R}\right)=0.049$} \\
\hline
\end{tabular}
simulated sample is initiated with 100 additional observations to get a stochastic initial state and these are then discarted. Estimates and Monte Carlo standard errors, reported in parentheses, are averages across simulations. UPPER \& LOWER PANEL: see caption of Table 1. 
Table III. Power of the 'J $\rightarrow$ LM' testing strategy when the data are generated by the LRE model in Eq.s (42)-(45) under indeterminacy.

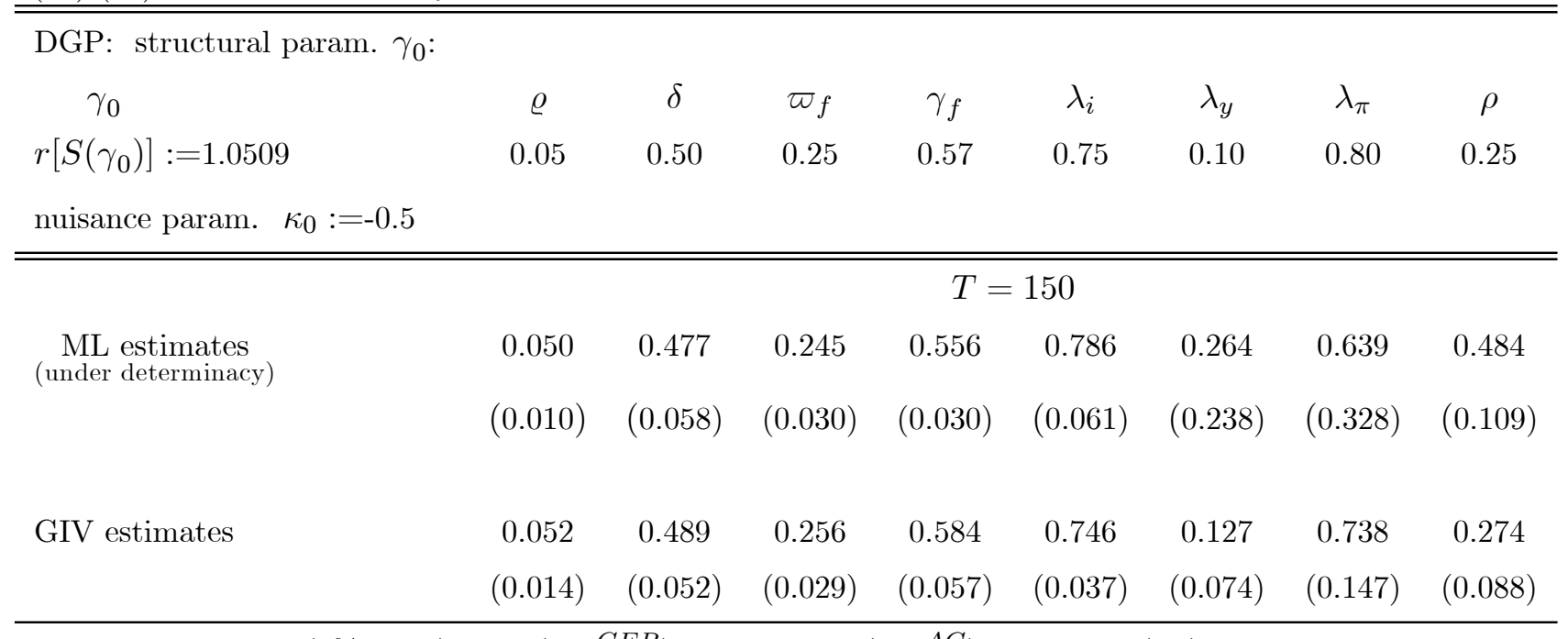

Marginal rej. freq. (5\% nom.) $\quad \operatorname{Rej}\left(\mathrm{LM}_{T}^{C E R}\right)=0.94, \quad \operatorname{Rej}\left(\mathrm{LM}_{T}^{A C}\right)=1, \quad \operatorname{Rej}\left(\mathrm{J}_{T}\right)=0.051$

Overall rej. freq. (5\% nom.) $\quad \operatorname{Rej}\left(\mathrm{J}_{T} \rightarrow \mathrm{LM}_{T}^{C E R}\right)=0.920$

$\operatorname{Rej}\left(\mathrm{J}_{T} \rightarrow \mathrm{LM}_{T}^{A C}\right)=0.999$

NOTES: Results are obtained using Monte Carlo simulations of $\mathrm{M}=1000$ samples of size $\mathrm{T}=150$.

Each simulated sample is initiated with 100 additional observations to get a stochastic initial state and these are then discarted. UPPER \& LOWER PANEL: see caption of Table 1. 
Table IV. Power of the 'J $\rightarrow$ LM' testing strategy when the data are generated by the LRE model in Eq.s (42)-(45) under indeterminacy $\left(H_{1}\right)$.

\begin{tabular}{lcccc}
\hline \hline DGP: $\gamma_{0}$ and $S\left(\gamma_{0}\right)$ as in Table III & \multicolumn{5}{l}{} \\
\hline \hline & $\operatorname{Rej}\left(\mathrm{LM}_{T}^{C E R}\right)$ & $\operatorname{Rej}\left(\mathrm{LM}_{T}^{A C}\right)$ & $\begin{array}{c}\operatorname{Rej}\left(\mathrm{J}_{T} \rightarrow \mathrm{LM}_{T}^{C E R}\right) \\
T=150\end{array}$ & $\left.\operatorname{Rej}_{T} \rightarrow \mathrm{J}_{T} \rightarrow \mathrm{LM}_{T}^{A C}\right)$ \\
$\kappa_{0}:=-0.5$ & 0.94 & 1 & 0.920 & 0.999 \\
$\kappa_{0}:=0.5$ & 0.858 & 0.384 & 0.817 & 0.267 \\
$\kappa_{0}:=-0.8$ & 0.954 & 1 & 0.944 & 1 \\
$\kappa_{0}:=0.8$ & 0.861 & 0.583 & 0.804 & 0.458 \\
$\kappa_{0}:=0.99$ & 0.139 & 0.055 & 0.106 & 0.03 \\
$\kappa_{0}:=-15$ & 0.975 & 0.367 & 0.971 & 0.257 \\
$\kappa_{0}:=15$ & 0.970 & 0.122 & 0.969 & 0.065 \\
$\kappa_{0}:=-0.5$ & & & $T=70$ & 0.788 \\
$\kappa_{0}:=0.5$ & 0.777 & 0.852 & 0.719 & 0.083 \\
$\kappa_{0}:=-0.8$ & 0.677 & 0.150 & 0.602 & 0.916 \\
$\kappa_{0}:=0.8$ & 0.861 & 0.947 & 0.820 & 0.085 \\
$\kappa_{0}:=0.99$ & 0.452 & 0.236 & 0.337 & 0.031 \\
$\kappa_{0}:=-15$ & & & & 0.912 \\
$\kappa_{0}:=15$ & 0.931 & 0.157 & 0.909 &
\end{tabular}

NOTES: Results are obtained using Monte Carlo simulations of $\mathrm{M}=1000$ samples of size $\mathrm{T}=150$ and $\mathrm{T}=70$, respectively. Each simulated sample is initiated with 100 additional observations to get a stochastic initial state and these are then discarted. GIV and ML estimates have been obtained as detailed in the caption of Table 1. Rej(·) stands for 'rejection frequency obtained at the $5 \%$ nominal significance level'. $\operatorname{Rej}(\cdot)$ stands for 'rejection frequency obtained at the $5 \%$ nominal significance level'; $5 \%$ is used for both the marginal tests and the joint test. For $\kappa_{0}:=15$ and $T=1500$ we obtain $\operatorname{Rej}\left(\mathrm{J}_{T} \rightarrow \operatorname{LM}_{T}^{A C}\right):=0.918$. 
Table V. Power of the ' $\mathrm{J} \rightarrow \mathrm{LM}$ ' testing strategy when the data are generated from the LRE model in Eq. (41) (dynamic misspecification, $H_{D M}$ ).

\begin{tabular}{|c|c|c|c|c|}
\hline \multicolumn{5}{|c|}{$\begin{array}{l}\text { DGP: system (41) with } \Gamma_{b, 3}:= \\
\text { Misspecification parameter } \mu:\end{array}$} \\
\hline & $\operatorname{Rej}\left(\mathrm{J}_{T}\right)$ & $\operatorname{Rej}\left(\mathrm{LM}_{T}^{C E R}\right)$ & $\operatorname{Rej}\left(\mathrm{J}_{T} \rightarrow \mathrm{LM}_{T}^{C E R}\right)$ & $\operatorname{Rej}\left(\mathrm{J}_{T} \rightarrow \mathrm{LM}_{T}^{A C}\right)$ \\
\hline & \multicolumn{4}{|c|}{$T=150$} \\
\hline$\mu_{0}:=-0.5$ & 1 & 1 & 1 & 1 \\
\hline$\mu_{0}:=-0.4$ & 0.990 & 1 & 0.994 & 0.983 \\
\hline$\mu_{0}:=-0.3$ & 0.789 & 1 & 0.962 & 0.723 \\
\hline$\mu_{0}:=-0.20$ & 0.260 & 0.762 & 0.621 & 0.200 \\
\hline \multirow[t]{2}{*}{$\mu_{0}:=-0.10$} & 0.07 & 0.122 & 0.092 & 0.053 \\
\hline & \multicolumn{4}{|c|}{$T=70$} \\
\hline$\mu_{0}:=-0.5$ & 0.970 & 1 & 0.989 & 0.954 \\
\hline$\mu_{0}:=-0.4$ & 0.786 & 0.988 & 0.739 & 0.710 \\
\hline$\mu_{0}:=-0.3$ & 0.414 & 0.857 & 0.590 & 0.326 \\
\hline$\mu_{0}:=-0.20$ & 0.147 & 0.367 & 0.226 & 0.109 \\
\hline$\mu_{0}:=-0.10$ & 0.058 & 0.110 & 0.078 & 0.044 \\
\hline
\end{tabular}

NOTES: Results are obtained using Monte Carlo simulations of $\mathrm{M}=1000$ samples of size $\mathrm{T}=150$ and $\mathrm{T}=70$, respectively. Each simulated sample is initiated with 100 additional observations to get a stochastic initial state and these are then discarted. Data are generated by a version of system (42)-(45) nested in in Eq. (41) with $\Gamma_{f, h}:=0_{3 \times 3}$ for $h \geq 2$ and $k_{1}:=3, \Gamma_{b, 3}:=\mu I_{3}$; all values of $\mu$ reported in the first column of Table 5 are such that the system has a determinate equilibrium. GIV and ML estimates have been obtained as detailed in the caption of Table 1 . Rej $(\cdot)$ stands for 'rejection frequency obtained at the $5 \%$ nominal significance level'. $\operatorname{Rej}(\cdot)$ stands for 'rejection frequency obtained at the $5 \%$ nominal significance level'; $5 \%$ is used for both the marginal tests and the joint test. 
Table VI. GIV and ML estimates of a small-scale DSGE model of the US economy and $\mathrm{J}_{T}$-LM $\mathrm{LM}_{T}$ test.

\begin{tabular}{|c|c|c|c|c|c|c|}
\hline & \multicolumn{2}{|c|}{$1960 \mathrm{q} 1-1979 \mathrm{q} 2(\mathrm{~T}=76)$} & \multicolumn{2}{|c|}{$1979 q 3-1997 q 4(\mathrm{~T}=74)$} & \multicolumn{2}{|c|}{$1960 \mathrm{q} 1-1997 \mathrm{q} 4 \quad(\mathrm{~T}=150)$} \\
\hline & GIV & ML & GIV & ML & GIV & ML \\
\hline$\varpi_{f}$ & $\begin{array}{c}0.761 \\
(0.138)\end{array}$ & $\begin{array}{c}0.629 \\
(0.264)\end{array}$ & $\begin{array}{c}0.631 \\
(0.153)\end{array}$ & $\begin{array}{c}0.805 \\
(0.147)\end{array}$ & $\begin{array}{c}0.694 \\
(0.126)\end{array}$ & $\begin{array}{c}0.508 \\
(0.182)\end{array}$ \\
\hline$\varkappa$ & $\begin{array}{c}0.954 \\
(0.374)\end{array}$ & $\begin{array}{l}-0.270 \\
(0.177)\end{array}$ & $\begin{array}{l}1.145 \\
(0.498)\end{array}$ & $\begin{array}{l}-0.092 \\
(0.271)\end{array}$ & $\begin{array}{c}0.887 \\
(0.330)\end{array}$ & $\begin{array}{l}-0.109 \\
(0.231)\end{array}$ \\
\hline$\lambda_{i}$ & $\begin{array}{c}0.795 \\
(0.077)\end{array}$ & $\begin{array}{c}0.831 \\
(0.082)\end{array}$ & $\begin{array}{c}0.899 \\
(0.029)\end{array}$ & $\begin{array}{c}0.931 \\
(0.059)\end{array}$ & $\begin{array}{c}0.879 \\
(0.041)\end{array}$ & $\begin{array}{c}0.906 \\
(0.049)\end{array}$ \\
\hline$\lambda_{y}$ & $\begin{array}{c}1.504 \\
(0.473)\end{array}$ & $\begin{array}{l}1.838 \\
(0.807)\end{array}$ & $\begin{array}{l}1.545 \\
(0.565)\end{array}$ & $\begin{array}{l}1.388 \\
(1.01)\end{array}$ & $\begin{array}{c}1.778 \\
(0.522)\end{array}$ & $\begin{array}{c}1.711 \\
(0.813)\end{array}$ \\
\hline$\lambda_{\pi}$ & $\begin{array}{c}0.619 \\
(0.329)\end{array}$ & $\begin{array}{c}0.498 \\
(0.288)\end{array}$ & $\begin{array}{c}0.960 \\
(0.315)\end{array}$ & $\begin{array}{c}0.491 \\
(0.497)\end{array}$ & $\begin{array}{c}0.737 \\
(0.386)\end{array}$ & $\begin{array}{c}0.00 \\
(0.334)\end{array}$ \\
\hline$\rho_{y}$ & $\begin{array}{c}0.888 \\
(0.105)\end{array}$ & $\begin{array}{c}0.892 \\
(0.146)\end{array}$ & $\begin{array}{c}0.997 \\
(0.061)\end{array}$ & $\begin{array}{c}0.974 \\
(0.083)\end{array}$ & $\begin{array}{c}0.965 \\
(0.073)\end{array}$ & $\begin{array}{c}0.874 \\
(0.083)\end{array}$ \\
\hline$\rho_{\pi}$ & $\begin{array}{c}0.494 \\
(0.216)\end{array}$ & $\begin{array}{c}0.931 \\
(0.171)\end{array}$ & $\begin{array}{c}0.520 \\
(0.334)\end{array}$ & $\begin{array}{c}0.585 \\
(0.148)\end{array}$ & $\begin{array}{c}0.424 \\
(0.205)\end{array}$ & $\begin{array}{c}0.833 \\
(0.175)\end{array}$ \\
\hline \multirow[t]{4}{*}{$\rho_{i}$} & $\begin{array}{c}0.178 \\
(0.136) \\
\end{array}$ & $\begin{array}{c}0.102 \\
(0.139) \\
\end{array}$ & $\begin{array}{l}-0.047 \\
(0.067) \\
\end{array}$ & $\begin{array}{l}-0.115 \\
(0.077) \\
\end{array}$ & $\begin{array}{c}0.052 \\
(0.095) \\
\end{array}$ & $\begin{array}{l}-0.077 \\
(0.086) \\
\end{array}$ \\
\hline & \multicolumn{2}{|c|}{$\mathrm{J}_{T}=\begin{array}{c}12.59 \\
{[0.182]}\end{array}$} & \multicolumn{2}{|c|}{$\begin{array}{c}\mathrm{J}_{T}=60.58 \\
{[0.000]}\end{array}$} & \multicolumn{2}{|c|}{$\mathrm{J}_{T}=\begin{array}{c}40.27 \\
{[0.000]}\end{array}$} \\
\hline & \multicolumn{2}{|c|}{$\begin{aligned} & \mathrm{LM}_{T}= 30.60 \\
& {[0.000] }\end{aligned}$} & \multicolumn{2}{|c|}{$\begin{array}{r}\mathrm{LM}_{T}= \\
{[0.000]}\end{array}$} & \multicolumn{2}{|c|}{$\begin{array}{r}\mathrm{LM}_{T}= \\
{[0.000]}\end{array}$} \\
\hline & \multicolumn{2}{|c|}{$r[S(\hat{\gamma})]=0.973$} & \multicolumn{2}{|c|}{$r[S(\hat{\gamma})]=1.003$} & & \\
\hline \multicolumn{5}{|c|}{ Break at 1979.q2: $\mathrm{D}_{T}=\begin{array}{c}5.75 \\
{[0.76]}\end{array}, \mathrm{O}_{T}=34.52$} & & \\
\hline
\end{tabular}

NOTES: Data are the same as in Lubik and Shorfheide (2004) and refer to the US economy. The parameter $\varkappa$ is related to $\gamma_{f}$ and $\gamma_{b}$ by $\gamma_{f}:=\beta /(1+\varkappa \beta)$ and $\gamma_{b}:=\varkappa /(1+\varkappa \beta)$, where the discount factor $\beta$ is set at 0.99 . GIV and ML estimates have been obtained as detailed in the caption of Table 1. $r[\cdot]$ is the spectral radius operator. Standard errors in parentheses; p-values in brackets. $\mathrm{D}_{T}$ is the 'D statistic' for a structural break in 1979q3 which affects the structural parameters alone (Hall, 2005, Ch.5). $\mathrm{O}_{T}$ is Hall and Sen's (1999) test for a structural break in 1979q3 which affects the overidentifying restrictions alone. 
Table VII. Further Monte Carlo results

\begin{tabular}{|c|c|c|c|c|}
\hline \multicolumn{2}{|c|}{ Frequency $\operatorname{Fr}[r[S(\hat{\gamma})]>1]$} & & & \\
\hline \multicolumn{5}{|l|}{ DGPs: } \\
\hline \multicolumn{2}{|c|}{ Table 1 of paper: } & \multicolumn{3}{|l|}{0.11} \\
\hline \multicolumn{2}{|c|}{ Table 2 of paper: } & \multicolumn{3}{|l|}{0.59} \\
\hline \multicolumn{2}{|c|}{ Table 4 of paper with $\kappa_{0}:=0.99$ : } & \multicolumn{3}{|l|}{0.63} \\
\hline \multicolumn{2}{|c|}{ Table 5 of paper with $\mu_{0}:=-0.5$} & \multicolumn{3}{|l|}{0.74} \\
\hline \multicolumn{3}{|c|}{ Table 5 of paper with $\mu_{0}:=-0.1 \quad 0.51$} & & \\
\hline \multicolumn{5}{|c|}{$\begin{array}{l}\text { then discarted. } \operatorname{Fr}[\cdot] \text { measures the number of times out ot M in which the spectral radion } \\
\hat{\gamma} \text { ) matrix is found to be greater than one, } \hat{\gamma} \text { being the roboust point GIV estimator of the st } \\
\text { cameters. }\end{array}$} \\
\hline \multicolumn{5}{|c|}{ Table VIII. Reduced form evidence on US data. } \\
\hline & \multicolumn{2}{|c|}{ Pre-Volcker (Period 1) } & \multicolumn{2}{|c|}{ Volcker-Greenspan (Period 2) } \\
\hline lags & 2 & 3 & 2 & 3 \\
\hline $\mathrm{AIC}$ & 7.97 & $7.77^{*}$ & 8.58 & $8.35^{*}$ \\
\hline HQ & 8.23 & $8.14^{*}$ & 8.84 & $8.73^{*}$ \\
\hline $\mathrm{SC}$ & $8.62^{*}$ & 8.70 & $9.24^{*}$ & 9.31 \\
\hline $\operatorname{dg}\left(\hat{\Sigma}_{\varepsilon}\right)^{\prime}$ & $(0.87,1.21,0.66)$ & $(0.86,1.16,0.60)$ & $(0.61,1.67,1.07)$ & $(0.58,1.48,1.01)$ \\
\hline Autocor. & $1.61[0.02]$ & $1.04[0.43]$ & $1.96[0.00]$ & $1.96[0.00]$ \\
\hline Normality & $14.24[0.03]$ & $13.26[0.04]$ & $25.00[0.00]$ & $25.82[0.00]$ \\
\hline largest root & 0.91 & 0.91 & 0.86 & 0.88 \\
\hline LR: 3 to 2 lags & \multicolumn{2}{|c|}{$33.49[0.00]$} & \multicolumn{2}{|c|}{$30.32[0.00]$} \\
\hline
\end{tabular}

NOTES: The VARs for $X_{t}:=\left(y_{t}, \pi_{t}, i_{t}\right)^{\prime}$ are estimated on the same data as Lubik and Shorfheide (2004). Estimation on each sub-period is carried out by considering within-periods initial values. AIC=Akaike; $\mathrm{HQ}=$ Hannan-Quinn; $\mathrm{SC}=$ Schwarz; ' $\mathrm{dg}\left(\hat{\Sigma}_{\varepsilon}\right)$ ' denotes the diagonal elements of the estimated VAR covariance matrix; 'Autocor' is a LM vector test for residual autocorrelation up to 5 lags; 'Normality' is a LM vector test for residual normality; 'largest root' is the estimated spectral radius of the VAR companion matrix; p-values in squared brackets. 